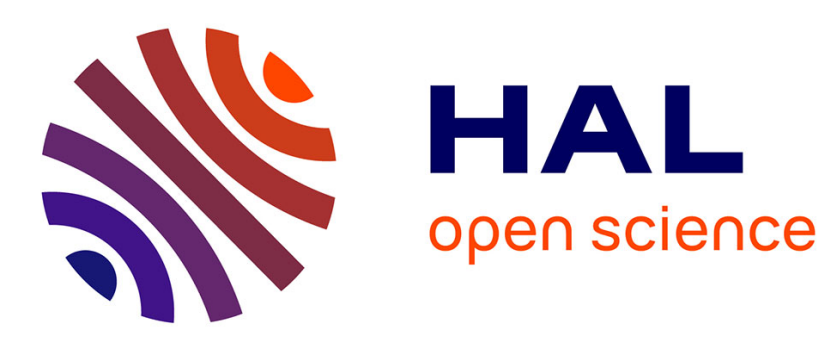

\title{
Enablers for robust POD models
}

Michel Bergmann, Charles-Henri Bruneau, Angelo Iollo

\section{To cite this version:}

Michel Bergmann, Charles-Henri Bruneau, Angelo Iollo. Enablers for robust POD models. Journal of

Computational Physics, 2009, 228 (2), pp.516-538. 10.1016/j.jcp.2008.09.024 . inria-00338203

\section{HAL Id: inria-00338203 \\ https://hal.inria.fr/inria-00338203}

Submitted on 12 Nov 2008

HAL is a multi-disciplinary open access archive for the deposit and dissemination of scientific research documents, whether they are published or not. The documents may come from teaching and research institutions in France or abroad, or from public or private research centers.
L'archive ouverte pluridisciplinaire HAL, est destinée au dépôt et à la diffusion de documents scientifiques de niveau recherche, publiés ou non, émanant des établissements d'enseignement et de recherche français ou étrangers, des laboratoires publics ou privés. 


\title{
Enablers for robust POD models
}

\author{
M. Bergmann ${ }^{\text {a,* }}$, C.-H. Bruneau ${ }^{\text {a }}$ A. Iollo ${ }^{\text {a }}$ \\ ${ }^{a}$ INRIA Bordeaux Sud Ouest, Team MC2 and Institut de Mathématiques de \\ Bordeaux, Université Bordeaux 1, UMR 5251 CNRS, 351, Cours de la Libération, \\ 33405 Talence cedex, France
}

\begin{abstract}
This paper focuses on improving the stability as well as the approximation properties of Reduced Order Models (ROM) based on Proper Orthogonal Decomposition (POD). The ROM is obtained by seeking a solution belonging to the POD subspace and that at the same time minimizes the Navier-Stokes residuals. We propose a modified ROM that directly incorporates the pressure term in the model. The $\mathrm{ROM}$ is then stabilized making use of a method based on the fine scale equations. An improvement of the POD solution subspace is performed thanks to an hybrid method that couples direct numerical simulations and reduced order model simulations. The methods proposed are tested on the two-dimensional confined square cylinder wake flow in laminar regime.
\end{abstract}

Key words: Proper Orthogonal Decomposition, Reduced Order Model, Stabilization, Functional subspace improvement

\section{INTRODUCTION}

\subsection{Reduced Order Models based on Proper Orthogonal Decomposition}

These last decades, the conception and the optimization of the aerodynamics/aeroacoustics of ground vehicles and airplanes has been pursued by numerical simulation. The applications mainly concern unsteady turbulent flows that develop at high Reynolds numbers. The numerical simulation of such flows, as well as their control, requires massive computational resources. Indeed, after discretization of the governing equations, i.e. the Navier-Stokes equations

* Corresponding author.

Email address: Michel.Bergmann@inria.fr (M. Bergmann). 
in fluid mechanics context, one must then solve a system of equations whose complexity algebraically grows with the number of degrees of freedom of the system to be solved. Now, and despite of the considerable progress made in the numerical field (power of the computers, new and more efficient algorithms), it is still very difficult to solve such large problems for complex flows in real time, that is, in fine, a major stake for industrials. To overcome this difficulty, it is possible to determine a reduced order model of the flow dynamics keeping only few adapted modes. The choice of these modes is not unique, and it strongly depends on the characteristics of the flow that one wants to approximate, or even might depend on some expected outputs (Goal-oriented models [1]). Several methods are commonly used, among them Proper Orthogonal Decomposition (POD) [2-4], balanced truncation [5-7], global eigenmodes [8], Galerkin modes [9] etc. Due to the energetic optimality of its basis, the POD is chosen in this study. By this technique it is possible to extract the dominant characteristics (POD modes) of a given database, and the ROM is then obtained thanks to a Galerkin projection of the governing equations onto these modes. Although this method for reducing the order of a system can be very efficient in some flow configurations, it also presents several drawbacks. Besides the possible inherent lack of numerical stability of POD/Galerkin methods, even for simple systems [10], the main shortcomings are the following:

- Since in most of the POD applications for incompressible flows the POD ROM is built from a velocity database ${ }^{1}$ it is necessary to model the pressure term. Usually, in many closed flows, the contribution of the pressure term formally drops out due to fortunate choices of boundary conditions in the POD ROM. However, for convectively unstable shear layers, as the mixing layer or the wake flow, it was proved in [11] that neglecting the pressure term may lead to large amplitude errors in the Galerkin model. Therefore, to accurately model such flows, the pressure term $[11,12]$ must be modeled. To overcome this difficulty, a pressure extended Reduced Order Model is introduced in $\S 3$, so that the pressure term can be directly approximated using the pressure POD mode.

- Due to the energetic optimality of the POD basis functions, few modes are sufficient to give a good representation of the kinetic energy of the flow $^{2}$. For model reduction purpose, we only keep these few modes that are associated to the large eddies of the flow (as the vortices of the Von Kármán street that usually develop behind bluff bodies). But since the main amount of viscous dissipation takes place in the small eddies represented by basis functions that are not taken into account, the leading ROM is not able to dissipate enough energy. It is then necessary to close the ROM by modeling the interaction between the calculated modes and the non

\footnotetext{
1 In almost all experimental works the pressure field is unavailable.

2 This is true for 2-D periodic laminar flows, but thousands of POD modes could be necessary to describe the fluctuation energy of a fully developed turbulent flow.
} 


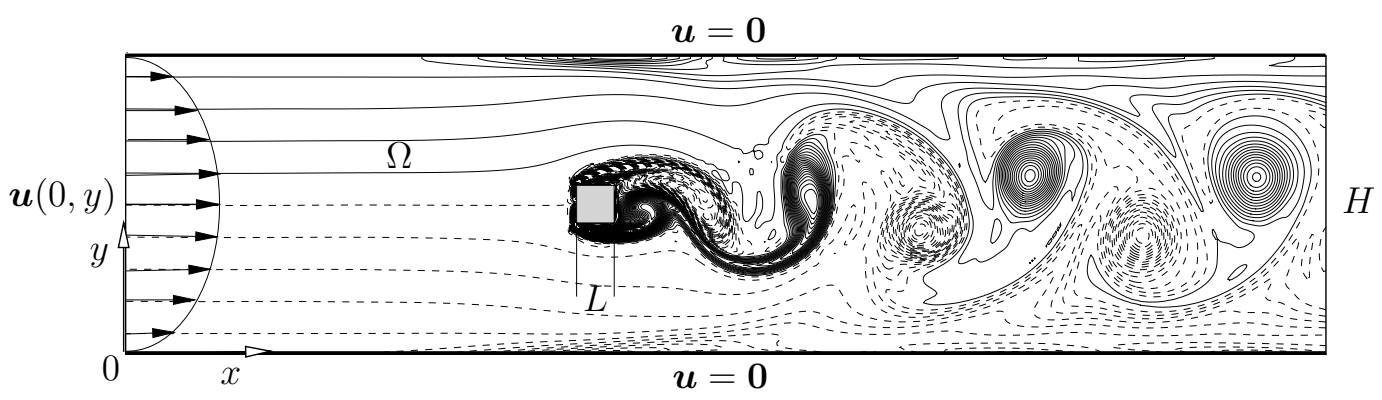

Fig. 1. Flow configuration and vorticity snapshot at $R e=200$.

resolved modes. This problem is similar to that of Large Eddy Simulation (LES) [13] of turbulent flows. In this study, the ROM is closed using NavierStokes equations residuals and exploiting ideas similar to Streamline Upwind Petrov-Galerkin (SUPG) and Variational Multiscale (VMS) methods [14].

- Since POD basis functions are optimal to represent the main characteristics included in the snapshot database of the flow configuration used to build them, the same basis functions are a priori not optimal to efficiently represent the main characteristics of other flow configurations. Indeed, for flow control purpose, it was demonstrated [15-17] that POD basis functions built from a flow database generated with a given set of control parameters is not able to represent the main features of a flow generated with another set of control parameters. To overcome this problem, we propose to derive methods allowing to adapt the POD basis functions at low numerical costs. This is the central question of $\S 5$.

\subsection{Flow configuration}

In this study the confined square cylinder wake flow (figure 1) is chosen as a prototype of separated flow. This flow is interesting since it presents detachments of the boundary layer, wake and vortices interactions with walls. The Navier-Stokes equations, written in their dimensionless and conservative form, write:

$$
\begin{aligned}
& \frac{\partial \boldsymbol{u}}{\partial t}+(\boldsymbol{u} \cdot \boldsymbol{\nabla}) \boldsymbol{u}=-\nabla p+\frac{1}{R e} \Delta \boldsymbol{u}, \\
& \boldsymbol{\nabla} \cdot \boldsymbol{u}=0,
\end{aligned}
$$

where $R e=U_{\infty} L / \nu$ denotes the Reynolds number, with $U_{\infty}=u(0, H / 2)$ the maximal inflow velocity, $L$ the lenght of the side of the square cylinder and $\nu$ the kinematic visosity. In what follows, we consider $R e=100$ and $R e=200$, that is to say, the laminar regime. Otherwise, the same parameters as those introduced in [12] are used in this study, i.e. the blockage ratio $\beta=L / H$ is equal to 0.125 and the domain $\Omega$ is $(0,4 H) \times(0, H)$. The same numerical method as that described in [12] is used. A vorticity representation of a flow 
snapshot is presented in figure 1 (dashed lines represent negative values). The boundary layer detachment, the wake and the vortex interactions with top and bottom walls are visible.

The paper is organized as follows. Section 2 presents the Proper Orthogonal Decomposition (POD, §2.1) and the standard velocity POD/Galerkin Reduced Order Model (POD ROM, §2.2) for incompressible flows. A pressure extended reduced order model is introduced in section 3. Different stabilization methods of the POD ROM are presented in section 4. A residuals based stabilization method (§4.1), and Streamline Upwind Petrov-Galerkin (SUPG) as well as the Variational Multiscale (VMS) methods ( $\$ 4.2)$ are introduced. Section 5 presents methods to adapt the functional subspace when input system parameters change. A Krylov like method ( $\$ 5.1)$ and an hybrid DNS/POD ROM method (§5.2) are presented. Finally, section 6 is dedicated to conclusions.

\section{Standard reduced order model based on proper orthogonal de- composition}

\subsection{Proper Orthogonal Decomposition}

The Proper Orthogonal Decomposition (POD) was first introduced in turbulence by Lumley [18] in 1967 as an unbiased definition of the coherent structures widely known to exist in a turbulent flow. A comprehensive review of the POD can be found in Refs [2-4]. The POD, also known as KarhunenLoève decomposition, principal component analysis or empirical eigenfunctions method, consists of looking for the deterministic function $\boldsymbol{\Phi}(\boldsymbol{x})$ that is most similar in an average sense to the realizations $\boldsymbol{U}(\boldsymbol{x}, t)$. For instance, the realizations $\boldsymbol{U}(\boldsymbol{x}, t)$ can be velocity fields, pressure fields, temperature fields, etc. Since in this study the data are issued from numerical simulations, the method to compute POD modes introduced by Sirovich [3] is adopted (see [4] for justifications). In this case, the constrained optimization problem reduces to the following Fredholm integral eigenvalue problem:

$$
\int_{0}^{T} C\left(t, t^{\prime}\right) a_{n}\left(t^{\prime}\right) \mathrm{d} t^{\prime}=\lambda_{n} a_{n}(t)
$$

where the temporal correlation tensor $C\left(t, t^{\prime}\right)$ is defined by:

$$
C\left(t, t^{\prime}\right)=\frac{1}{T}\left(\boldsymbol{U}(\boldsymbol{x}, t), \boldsymbol{U}\left(\boldsymbol{x}, t^{\prime}\right)\right)_{\Omega}
$$


The inner product $(., .)_{\Omega}$ between two fields $\boldsymbol{U}$ and $\boldsymbol{V}$ is computed as:

$$
(\boldsymbol{U}, \boldsymbol{V})_{\Omega}=\int_{\Omega} \boldsymbol{U} \cdot \boldsymbol{V} \mathrm{d} \boldsymbol{x}=\int_{\Omega} \sum_{i=1}^{n_{c}} U^{i} V^{i} \mathrm{~d} \boldsymbol{x},
$$

where $U^{i}$ represents the $i^{\text {th }}$ component of the vector $\boldsymbol{U}$ with dimension $n_{c}$.

The eigenvalues $\lambda_{n}(n=1,2, \ldots)$ determined in (2) are all real and positive and form a decreasing and convergent series. Each eigenvalue represents the contribution of the corresponding mode $\boldsymbol{\Phi}_{n}$ to the information content of the original data. Note that if $\boldsymbol{U}$ are the velocity fields, the information content reduces to the kinetic energy.

In Eq. (2), $a_{n}$ are the time-dependent POD eigenfunctions of order $n$. These modes form an orthogonal set, satisfying the condition:

$$
\frac{1}{T} \int_{0}^{T} a_{n}(t) a_{m}(t) \mathrm{d} t=\lambda_{n} \delta_{n m}
$$

The associated eigenvectors $\boldsymbol{\Phi}_{n}$ (also called empirical eigenfunctions) form a complete orthogonal set and are normalized, so that they verify $\left(\boldsymbol{\Phi}_{n}, \boldsymbol{\Phi}_{m}\right)_{\Omega}=$ $\delta_{n m}$.

The spatial basis functions $\Phi_{n}^{i}$ can then be calculated from the realizations $U^{i}$ and the coefficients $a_{n}$ with:

$$
\Phi_{n}^{i}(\boldsymbol{x})=\frac{1}{T \lambda_{n}} \int_{0}^{T} U^{i}(\boldsymbol{x}, t) a_{n}(t) \mathrm{d} t .
$$

Since the POD eigenfunctions can be represented as linear combinations of the realizations, they inherit all the properties of the original data. For instance, the eigenfunctions are divergence free for incompressible flows. Moreover, the eigenfunctions verify the boundary conditions of the numerical simulation used to determine the flow realizations.

The set of POD modes $\left\{\boldsymbol{\Phi}_{n}\right\}_{n=1}^{N_{P O D}}$ is complete in the sense that any realization $\boldsymbol{U}(\boldsymbol{x}, t)$ contained in the original data set, can be expanded with arbitrary accuracy (in function of $N_{P O D} \geq 1$ ) in the eigenfunctions as

$$
\boldsymbol{U}(\boldsymbol{x}, t) \simeq \widehat{\boldsymbol{U}}^{\left[1, \cdots, N_{P O D}\right]}(\boldsymbol{x}, t)=\sum_{n=1}^{N_{P O D}} a_{n}(t) \boldsymbol{\Phi}_{n}(\boldsymbol{x}) .
$$

For later convenience, the estimation $\widehat{\boldsymbol{U}}^{\left[1, \cdots, N_{P O D}\right]}$ of $\boldsymbol{U}$ is introduced, where the brackets contain the indices of all employed modes. Hereafter, we consider that the ensemble used to determine the POD modes consists of $N_{s}$ flow realizations (called time snapshots) $\boldsymbol{U}\left(\boldsymbol{x}, t_{i}\right), \boldsymbol{x} \in \Omega$, taken at $t_{i} \in[0, T], i=1, \cdots, N_{t}$. 
The energetic optimality of the POD basis functions suggests that only a very small number of POD modes may be necessary to describe efficiently any flow realizations of the input data i.e. $N_{r} \ll N_{s}$. In practice, $N_{r}$ is usually determined as the smallest integer $M$ such that the Relative Information Content, $R I C(M)=\sum_{i=1}^{M} \lambda_{i} / \sum_{i=1}^{N_{s}} \lambda_{i}$, is greater than a predefined percentage of energy, $\delta$. So that $N_{P O D}=N_{r}$, and the approximation (6) becomes

$$
\boldsymbol{U}(\boldsymbol{x}, t) \simeq \widehat{\boldsymbol{U}}^{\left[1, \cdots, N_{r}\right]}(\boldsymbol{x}, t)=\sum_{n=1}^{N_{r}} a_{n}(t) \boldsymbol{\Phi}_{n}(\boldsymbol{x})
$$

\subsection{Classical reduced order model and drawbacks}

To derive a classical reduced order model only the velocity fields are used, so that $\boldsymbol{U}(\boldsymbol{x}, t) \equiv \boldsymbol{u}(\boldsymbol{x}, t)$. Thus, decomposition (7) becomes:

$$
\boldsymbol{u}(\boldsymbol{x}, t) \simeq \sum_{n=1}^{N_{r}} a_{n}(t) \phi_{n}(\boldsymbol{x})
$$

where $\phi_{n}$ denote the velocity POD basis functions. A low dimensional dynamical system is obtained via a Galerkin projection of the Navier-Stokes equations (1). The Galerkin projection formally writes:

$$
\left(\phi_{i}, \frac{\partial \boldsymbol{u}}{\partial t}+(\boldsymbol{u} \cdot \boldsymbol{\nabla}) \boldsymbol{u}\right)_{\Omega}=-\left(\phi_{i}, \nabla p\right)_{\Omega}+\left(\phi_{i}, \frac{1}{R e} \Delta \boldsymbol{u}\right)_{\Omega} .
$$

Note that since the pressure term $\left(\phi_{i}, \nabla p\right)_{\Omega}$ can not be evaluated using the standard velocity POD formulation, it is usually neglected (see discussion below). After some algebraic manipulations using decomposition (8), the reduced order model writes (see [19] for more details):

$$
\frac{\mathrm{d} a_{i}(t)}{\mathrm{d} t}=\mathcal{A}_{i}+\sum_{j=1}^{N_{r}} \mathcal{B}_{i j} a_{j}(t)+\sum_{j=1}^{N_{r}} \sum_{k=1}^{N_{r}} \mathcal{C}_{i j k} a_{j}(t) a_{k}(t) \quad i=1, \cdots, N_{r} .
$$

with initial conditions

$$
a_{i}(0)=\left(\boldsymbol{u}(\boldsymbol{x}, 0), \phi_{i}(\boldsymbol{x})\right)_{\Omega} \quad i=1, \cdots, N_{r}
$$

It is well known that when equations (10) are integrated in time a gradual drifting from the full-state solution to another erroneous state may arise after several vortex shedding periods, precluding a correct description of the longterm dynamics [20]. Even worse, in some cases, the short-term dynamics of the POD ROM may not be sufficiently accurate to be used as a surrogate model of the original high-fidelity model. Essentially, three sources of numerical errors can be identified. As it was already mentioned, the POD/Galerkin 
method can first present a lack of inherent numerical stability even for very simple problems [10]. Secondly, the pressure term is often neglected in the POD ROM. It is possible to model this term, but to avoid this modelisation, a pressure extended Reduced Order Model is introduced in $\S 3$. The third source of instability is the truncation involved in the POD-Galerkin approach. Indeed, since only the most energetic POD modes are kept, the POD ROM is not sufficiently dissipative to prevent erroneous time amplifications of its solution. This problem is similar to that of Large Eddy Simulation where the energy transfers between the resolved scales and the subgrid scales have to be modelled [13]. For instance, 4 modes are sufficient to restore more than $99 \%$ of the kinetic energy of the circular cylinder wake flow ( $2 D$, laminar regime), but the solution of the such reduced order model does not converge towards the numerical solution of the Navier-Stokes equations [21]. It is thus necessary to stabilize the POD ROM. In this study, thanks to the pressure extended reduced order model, the POD ROM can be stabilized using the Navier-Stokes operator residuals evaluated with the POD flow fields reconstructions (§4).

\section{A pressure extended Reduced Order Model}

It is demonstrated that the contribution of the pressure term vanishes in many closed flows. However, Noack [11] proved that neglecting the pressure term for convectively unstable shear layers (as the mixing layer or the wake flow) can lead to large amplitude errors in the Galerkin model. A solution is to model this pressure term $[11,12]$. One aim of this study is to invoke the least modelisation as possible. The purpose of this section is thus to derive a pressure extended Reduced Order Model, i.e. a ROM that allows to build both the velocity and the pressure fields. The pressure term can thus be easily calculated using $p=\widetilde{p}$ (see decomposition (11b)). Another key issue is that, knowing the pressure field, it is possible to evaluate the Navier-Stokes residuals ${ }^{3}$. Indeed, the Navier-Stokes residuals will be used to both stabilize $(\S 4)$ the ROM and to improve $(\S 5)$ the POD subspace.

\subsection{Construction of the pressure extended POD ROM}

As it was mentioned in $\S 2.2$, reduced order modeling is based on the restriction of the weak form of the Navier-Stokes equations to the subspace $\mathcal{S}_{N_{r}}^{P O D}$ spanned by the first $N_{r}$ spatial eigenfunctions $\boldsymbol{\Phi}_{i}$. Here, we develop a global basis for both the velocity and pressure fields (see [22] for justification and numerical

\footnotetext{
$\overline{3}$ Only the velocity field is necessary to evaluate the residuals of the Navier-Stokes operator written in its vorticity formulation.
} 
demonstration). The exact flow fields $\boldsymbol{u}$ and $p$ are then approximated by:

$$
\begin{gathered}
\widetilde{\boldsymbol{u}}(\boldsymbol{x}, t)=\sum_{i=1}^{N_{r}} a_{i}(t) \phi_{i}(\boldsymbol{x}) \\
\widetilde{p}(\boldsymbol{x}, t)=\sum_{i=1}^{N_{r}} a_{i}(t) \psi_{i}(\boldsymbol{x}) .
\end{gathered}
$$

The velocity and the pressure basis functions, $\phi_{i}$ and $\boldsymbol{\psi}_{i}$ respectively, are determined using $\boldsymbol{U}(\boldsymbol{x}, t)=(\widetilde{\boldsymbol{u}}(\boldsymbol{x}, t), \widetilde{p}(\boldsymbol{x}, t))^{T}$ to calculate the temporal correlation tensor (3). The basis functions $\boldsymbol{\phi}_{i}$ and $\boldsymbol{\psi}_{i}$ are determined as $\boldsymbol{\Phi}(\boldsymbol{x}, t)=$ $(\boldsymbol{\phi}(\boldsymbol{x}, t), \psi(\boldsymbol{x}, t))^{T}, \boldsymbol{\Phi}(\boldsymbol{x}, t)$ being obtained from (5).

The substitution of equations (11) in the Navier-Stokes momentum equations (1a) leads to:

$$
\sum_{j=1}^{N_{r}} \frac{\mathrm{d} a_{j}}{\mathrm{~d} t} \boldsymbol{\phi}_{j}+\left(\sum_{j=1}^{N_{r}} a_{j} \boldsymbol{\phi}_{j} \cdot \boldsymbol{\nabla}\right) \sum_{k=1}^{N_{r}} a_{k} \boldsymbol{\phi}_{k}=-\sum_{j=1}^{N_{r}} a_{j} \boldsymbol{\nabla} \psi_{j}+\frac{1}{R e} \sum_{j=1}^{N_{r}} a_{j} \Delta \boldsymbol{\phi}_{j},
$$

that is:

$$
\sum_{j=1}^{N_{r}} \boldsymbol{\phi}_{j} \frac{\mathrm{d} a_{j}}{\mathrm{~d} t}+\sum_{j=1}^{N_{r}} \sum_{k=1}^{N_{r}}\left(\boldsymbol{\phi}_{j} \cdot \boldsymbol{\nabla}\right) \boldsymbol{\phi}_{k} a_{j} a_{k}=-\sum_{j=1}^{N_{r}} \nabla \psi_{j} a_{j}+\frac{1}{R e} \sum_{j=1}^{N_{r}} \Delta \boldsymbol{\phi}_{j} a_{j} .
$$

A Galerkin projection of the momentum equations (13) yields:

$$
\left(\boldsymbol{\phi}_{i}, \sum_{j=1}^{N_{r}} \boldsymbol{\phi}_{j} \frac{\mathrm{d} a_{j}}{\mathrm{~d} t}+\sum_{j=1}^{N_{r}} \sum_{k=1}^{N_{r}}\left(\boldsymbol{\phi}_{j} \cdot \boldsymbol{\nabla}\right) \boldsymbol{\phi}_{k} a_{j} a_{k}+\sum_{j=1}^{N_{r}} \nabla \psi_{j} a_{j}-\frac{1}{R e} \sum_{j=1}^{N_{r}} \Delta \boldsymbol{\phi}_{j} a_{j}\right)_{\Omega}=0 .
$$

The Reduced Order Model is then:

$$
\sum_{j=1}^{N_{r}} L_{i j}^{(m)} \frac{\mathrm{d} a_{j}}{\mathrm{~d} t}=\sum_{j=1}^{N_{r}} B_{i j}^{(m)} a_{j}+\sum_{j=1}^{N_{r}} \sum_{k=1}^{N_{r}} C_{i j k}^{(m)} a_{j} a_{k},
$$

where the coefficients ${ }^{4} L_{i j}^{m}, B_{i j}^{m}$ and $C_{i j k}^{m}$ are given by:

$$
\begin{aligned}
& L_{i j}^{(m)}=+\left(\boldsymbol{\phi}_{i}, \boldsymbol{\phi}_{j}\right)_{\Omega}, \\
& B_{i j}^{(m)}=-\left(\boldsymbol{\phi}_{i}, \frac{1}{R e} \Delta \boldsymbol{\phi}_{j}-\nabla \psi_{j}\right)_{\Omega}, \\
& C_{i j k}^{(m)}=-\left(\boldsymbol{\phi}_{i},\left(\boldsymbol{\phi}_{j} \cdot \nabla\right) \boldsymbol{\phi}_{k}\right)_{\Omega} .
\end{aligned}
$$

Here the superscript $m$ stands for momentum equations.

$\overline{4}$ In a general way, we have $\left(\boldsymbol{\Phi}_{i}, \boldsymbol{\Phi}_{j}\right)_{\Omega}=\delta_{i j}$, but not $\left(\phi_{i}, \phi_{j}\right)_{\Omega}=\delta_{i j}$. So, $L_{i j}^{(m)} \neq \delta_{i j}$. 
In this reduced order model we used global basis functions built by POD, but this methodology could be transposed to other modal decompositions such as decomposition onto stability modes [8]. Moreover it could be interesting to use non divergence free modes, as Navier-Stokes residuals modes. Such modes can be used to stabilize $(\S 4)$ and to improve $(\S 5)$ the POD ROM. Hence, if model (15) is built using non divergence free modes, it does not satisfy the continuity equation (mass conservation). It is thus necessary to add a constraint in the reduced order model.

A modified ROM that satisfies both momentum and continuity equations can be obtained starting from the weak form of the Navier Stokes equations:

$$
\left(\boldsymbol{w}_{i}, \frac{\partial \boldsymbol{u}}{\partial t}+\nabla \cdot(\boldsymbol{u} \otimes \boldsymbol{u})+\nabla p-\frac{1}{R e} \Delta \boldsymbol{u}\right)_{\Omega}+\left(q_{i}, \boldsymbol{\nabla} \cdot \boldsymbol{u}\right)_{\Omega}=0
$$

where $\boldsymbol{w}_{i}$ and $q_{i}$ belong to appropriate functional spaces. The velocity and pressure fields are expanded onto the POD basis functions $\left\{\phi_{i}\right\}_{i=1}^{N_{r}}$ and $\left\{\psi_{i}\right\}_{i=1}^{N_{r}}$ using equations (11a) and (11b), respectively.

One approach is then to use a Galerkin projection where $\boldsymbol{w}_{i}=\phi_{i}$ and $q_{i}=\psi_{i}$ as done before (equation (14)). Another approach is to use $\boldsymbol{w}_{i}=\boldsymbol{\phi}_{i}$ and $q_{i}=\alpha\left(\boldsymbol{\nabla} \cdot \boldsymbol{\phi}_{i}\right)^{T}$. This choice would correspond to the minimization of the continuity residuals, $\sum_{j=1}^{N_{r}} a_{j} \boldsymbol{\nabla} \cdot \boldsymbol{\phi}_{j}$, in a least squares sense, so that in limit of large $\alpha$ we have:

$$
\sum_{j=1}^{N_{r}} B_{i j}^{c} a_{j}=0
$$

where $B_{i j}^{(c)}=\left(\boldsymbol{\nabla} \cdot \boldsymbol{\phi}_{i}\right)^{T} \boldsymbol{\nabla} \cdot \boldsymbol{\phi}_{j}$ and the superscript $c$ stands for continuity equation. Numerically, this second approach gives better results, and the modified ROM that we use is thus:

$$
\sum_{j=1}^{N_{r}} L_{i j}^{(m)} \frac{\mathrm{d} a_{j}}{\mathrm{~d} t}=\sum_{j=1}^{N_{r}}\left(B_{i j}^{(m)}+\alpha B_{i j}^{(c)}\right) a_{j}+\sum_{j=1}^{N_{r}} \sum_{k=1}^{N_{r}} C_{i j k}^{(m)} a_{j} a_{k}
$$

where the weight $\alpha$ has to be fixed. In this study, we chose $\alpha=10^{-2}$.

Since we use the flow-field decompositions (11) the mean flow is solved by the reduced order model. The mean flow is then $\overline{\boldsymbol{U}}(\boldsymbol{x}, t)=a_{1}(t) \boldsymbol{\Phi}_{1}(\boldsymbol{x})$. It is well known that a small drift of the first temporal coefficient $a_{1}$ can occur. The flow rate is thus modified. In order to keep the flow rate as constant, another constraint must be enforced in the reduced order model (18). For the 2D confined flow, the conservation of flow rate writes:

$$
\int_{\mathcal{S}} u \mathrm{~d} s=c,
$$

where $\mathcal{S}$ is a cross section of the channel and $c$ is a constant. For instance $\mathcal{S}$ could be the inflow or outflow height $H$ of the channel, or, at the abscissa of 
the cylinder with height $L, \mathcal{S}=H-L$ (see figure 1). Numerically, the flow rate has to be constant over each slice $\mathcal{S}_{l} \equiv \mathcal{S}\left(x_{l}\right), 1 \leq l \leq N_{X}$ where $N_{X}$ is the number of discretisation points in the $x$-direction (a cartesian mesh is used).

Denoting $\phi=\left(\phi^{u}, \phi^{v}\right)^{T}$ and using (11a), condition (19) is approximated by ${ }^{5}$ :

$$
\sum_{i=1}^{N_{r}} a_{j}(t) \int_{\mathcal{S}_{l}} \phi_{j}^{u} \mathrm{~d} s=c
$$

The constant is initially evaluated by projection of a given snapshot onto the basis functions $\phi$. Numerically, in this study we have $c \equiv 1$. The flow rate conservation writes:

$$
\sum_{j=1}^{N_{r}} \frac{\mathrm{d} a_{j}}{\mathrm{~d} t} \int_{\mathcal{S}_{l}} \boldsymbol{\phi}_{j}^{u} \mathrm{~d} s=0 .
$$

Denoting by $\boldsymbol{f}_{j}$ the vector with components $f_{j}^{l}=\int_{\mathcal{S}_{l}} \boldsymbol{\phi}_{j}^{u} \mathrm{~d} s$, the flow rate conservation over the whole domain $\Omega$ writes:

$$
\sum_{j=1}^{N_{r}} \frac{\mathrm{d} a_{j}}{\mathrm{~d} t} \boldsymbol{f}_{j}=\mathbf{0}
$$

These additional constraints are now taken into account by enlarging the projection space with $\beta \boldsymbol{f}_{i}$. In the limit of large $\beta$ we have in a least square sense:

$$
\sum_{j=1}^{N_{r}} L_{i j}^{r} \frac{\mathrm{d} a_{j}}{\mathrm{~d} t}=0
$$

where $L_{i j}^{r}=\boldsymbol{f}_{i}^{T} \boldsymbol{f}_{j}$. The superscript $r$ stands for flow rate conservation. Then, the reduced order model writes:

$$
\sum_{j=1}^{N_{r}}\left(L_{i j}^{(m)}+\beta L_{i j}^{(r)}\right) \frac{\mathrm{d} a_{j}}{\mathrm{~d} t}=\sum_{j=1}^{N_{r}}\left(B_{i j}^{(m)}+\alpha B_{i j}^{(c)}\right) a_{j}+\sum_{j=1}^{N_{r}} \sum_{k=1}^{N_{r}} C_{i j k}^{(m)} a_{j} a_{k}
$$

with initial conditions

$$
a_{i}(0)=\left(\boldsymbol{U}(\boldsymbol{x}, 0), \Phi_{i}(\boldsymbol{x})\right)_{\Omega} \quad i=1, \cdots, N_{r},
$$

where the weight $\beta$ has to be fixed. In this study, we chose $\beta=10^{2}$. This reduced order model satisfies the momentum equations, the continuity equation as well as the conservation of the flow rate, even for non divergence free modes.

$\overline{5}$ If one uses only POD modes, we can taken $N_{r}=1$ since the flow rate is only given by the mean flow. However, using other modes that do not respect a priori the flow rate conservation (as the residual modes), $N_{r} \neq 1$. 


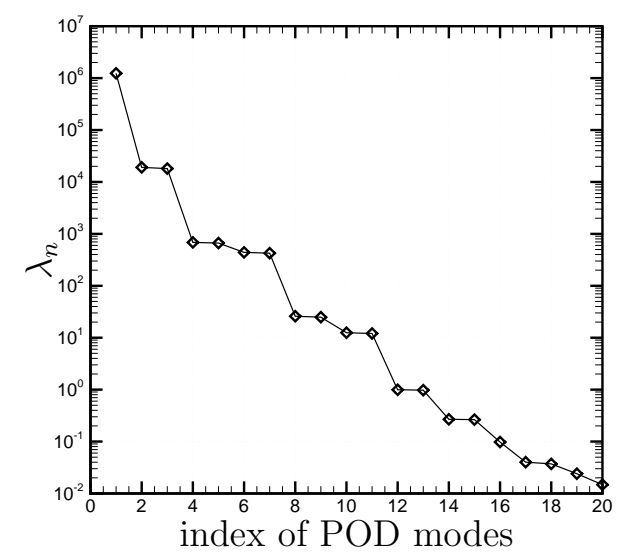

Fig. 2. Eigenvalues spectrum.

\subsection{Numerical results of the pressure extended POD ROM}

The reduced order model (21) is tested on a $2 \mathrm{D}$ confined square cylinder wake flow in laminar regime $(R e=200)$. In this section the POD basis $\boldsymbol{\Phi}$ is built following the POD snapshot method introduced by Sirovich [3]. Here, 80 snapshots uniformly distributed over one vortex shedding period are used to compute the discrete form of the temporal tensor (3). The corresponding eigenvalues spectrum is presented in Figure 2. This spectrum is degenerate presenting pairs of identical eigenvalues for the fluctuating modes (the mean flow is indexed by 1). The POD basis functions are obtained via a projection of the temporal tensor eigenvectors on the whole set of snapshots. Some of them are presented in figure 3 in terms of iso-vorticity (noted $\nabla_{\wedge} \phi_{i}$, for velocity modes $\phi_{i}$ ) and isobars (for pressure modes $\psi_{i}$ ). The evolution of the $R I C$ introduced in $\S 2.1$ is presented in Figure 4. Only the first 5 modes are sufficient to represent more than $98 \%$ of the total kinetic energy. However, another 5modes reduced order basis containing approximatively the same percentage of energy could be derived using modes 6 and 7 instead of 4 and 5. Indeed, even if these two pairs of modes are very different (see for instance the topological differences between $\phi_{5}$ and $\phi_{7}$ in Fig. 3), they have approximatively the same energetic contribution as one can see in Figure 5 where the Individual Enegetic Contribution (IEC) is presented. Thus, a judicious choice of the POD modes is not so evident in this case. Instead of using the RIC criterium, one can decide to keep all the fluctuating modes presenting an energy contribution greater than a given threshold (see Fig. 5). Here, all the modes with an energy contribution greater than $10^{-2}$ are kept. This corresponds to 10 fluctuating modes plus the mean flow mode, i.e. $N_{r}=11$ modes.

After having computed once the operators of the reduced order model (21) using these $N_{r}=11$ modes, a long time flow prediction over more than 1000 vortex shedding periods is performed. Figure 6 presents the temporal evolu- 

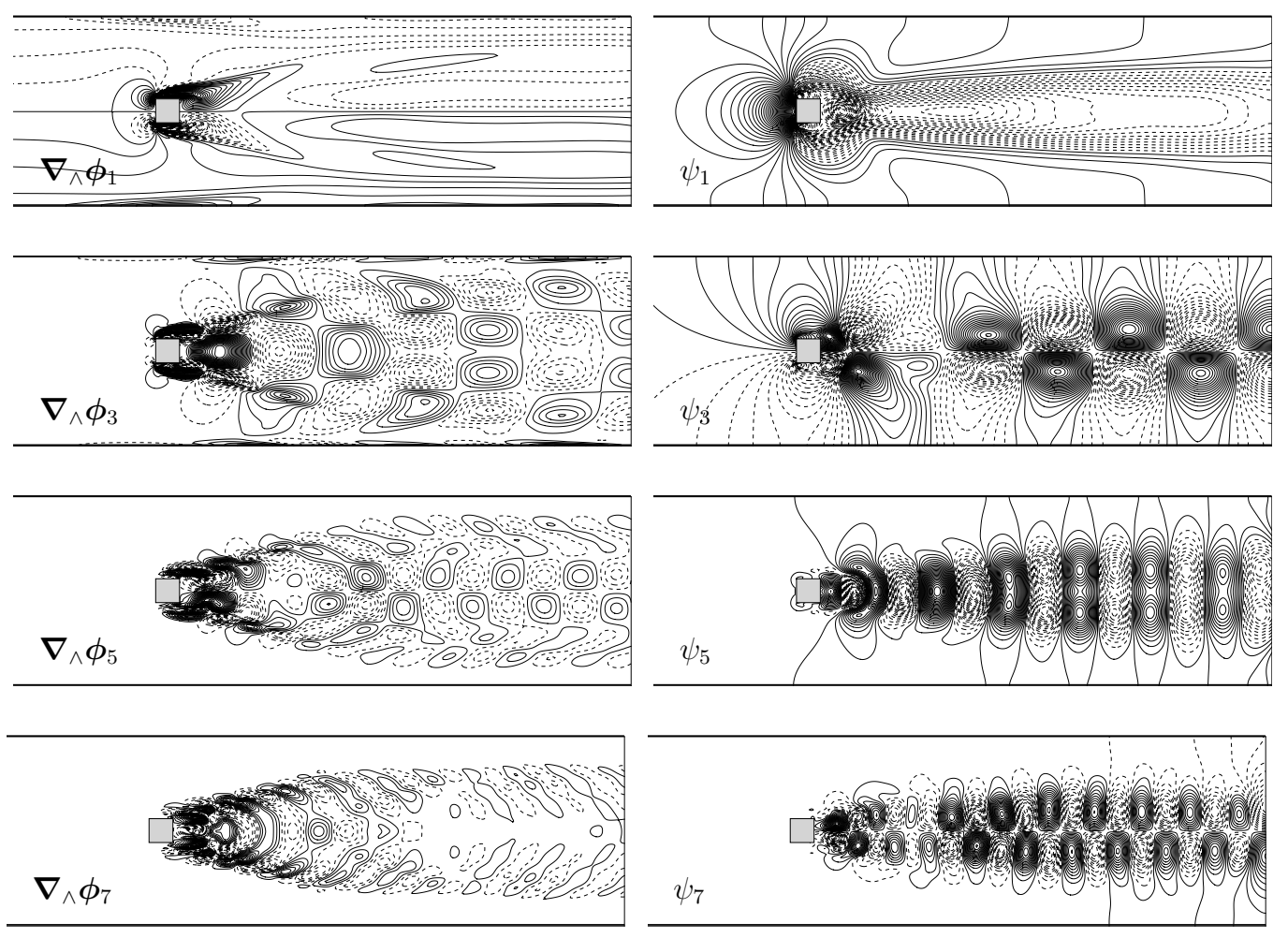

Fig. 3. Representation of some POD modes. Iso-vorticity (left) and isobars (right). Dashed lines represent negative values (the pressure reference is arbitrarily chosen to be zero).

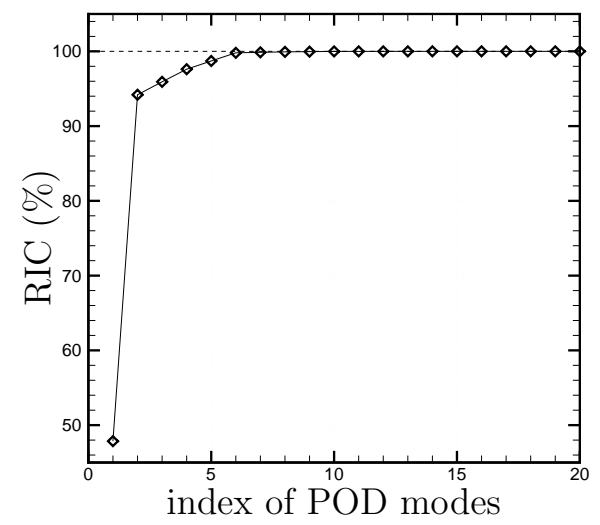

Fig. 4. RIC of fluctuating modes.

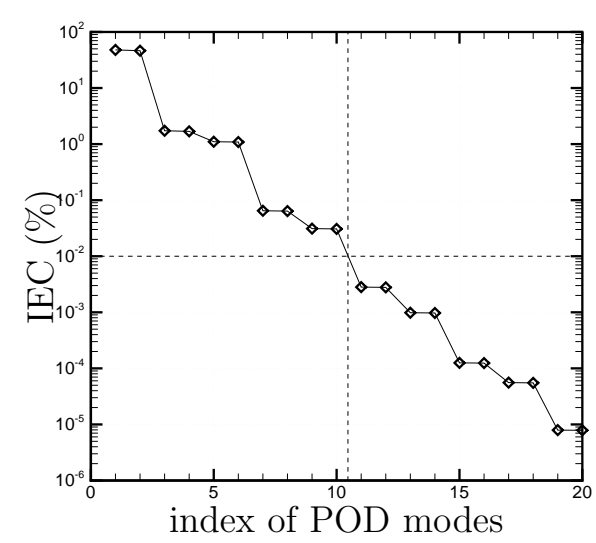

Fig. 5. Eigenvalues spectrum.

tion of the set of coefficients $\left\{a_{i}\right\}_{i=1}^{N_{r}}$, solution of the system (21), over the 40 vortex shedding periods. Since the temporal tensor eigenvalues spectrum is degenerate (it presents pairs of eigenvalues) only the odd-indexed coefficients are presented (the even ones have the same behavior). It is noticeable that no divergence occur for the long time prediction. A comparison of the projected (projection of the Navier-Stokes solution onto the POD basis) and 

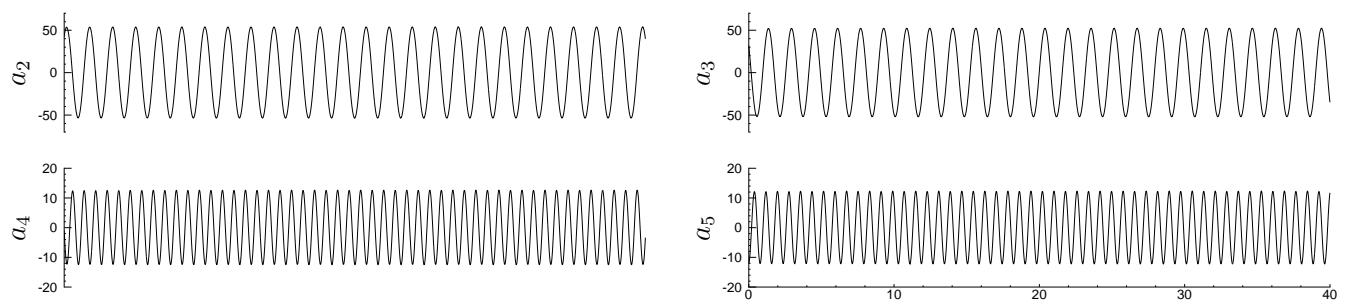

Fig. 6. Temporal evolutions of the predicted POD coefficients over 40 vortex shedding periods. 11 modes model.
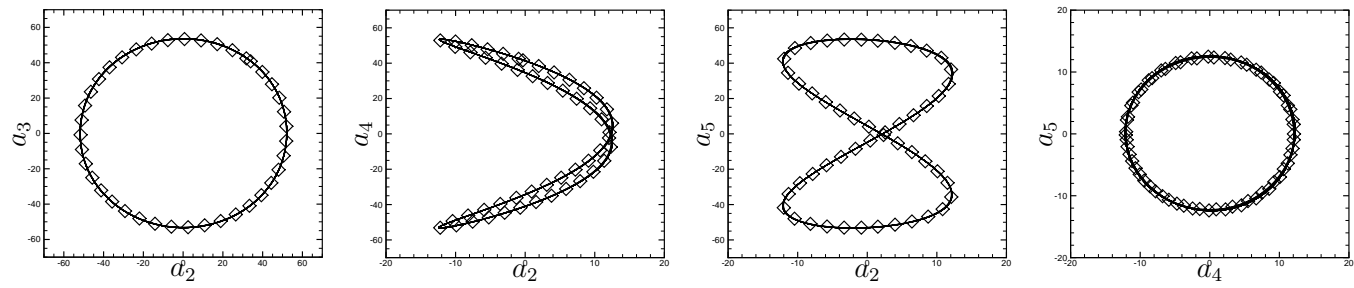

Fig. 7. Comparison of the projected (NS: $\diamond)$ and the predicted (ROM: - ) limit cycles over 1000 vortex shedding periods. 11 modes model.
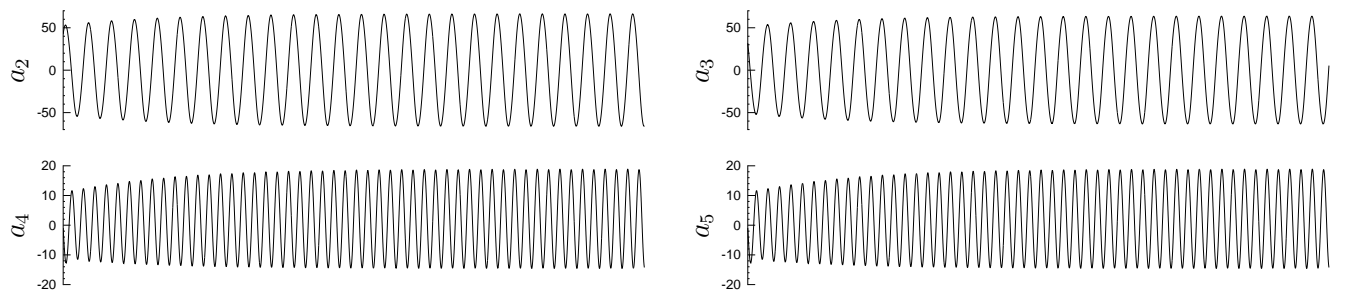

Fig. 8. Temporal evolutions of the predicted POD coefficients over 40 vortex shedding periods. 5 modes model.

the predicted (solutions of the ROM (21)) limit cycles over 1000 vortex shedding periods is presented in Figure 7 . The predicted limit cycles perfectly match the projected ones even for small scales (high order modes) where the high-frequency dynamics is more complex. The good accuracy for all limit cycles indicates also that no spurious dephasing occurs between modes. The system (21) with 11 modes is numerically stable for short and long time predictions so that no calibration procedures are needed. We want to highlight that a small gradual drifting could be observed using classical POD reduced order model where the pressure term remains unmodeled. It is thus important to calculate, or at least to model, the pressure term.

As it was already mentioned ( $(2.2)$, when system (21) is integrated in time a gradual drifting from the full-state solution to another erroneous state may arise after several vortex shedding periods if only a very small number of modes are kept. Indeed as it was shown in figures 8 and 9, the solution of model (21) built with 5 modes reaches erroneous limit cycles, and can even diverge with 3 modes (see figures 10 and 11). In this simple test case, only $N_{r}=11$ modes 

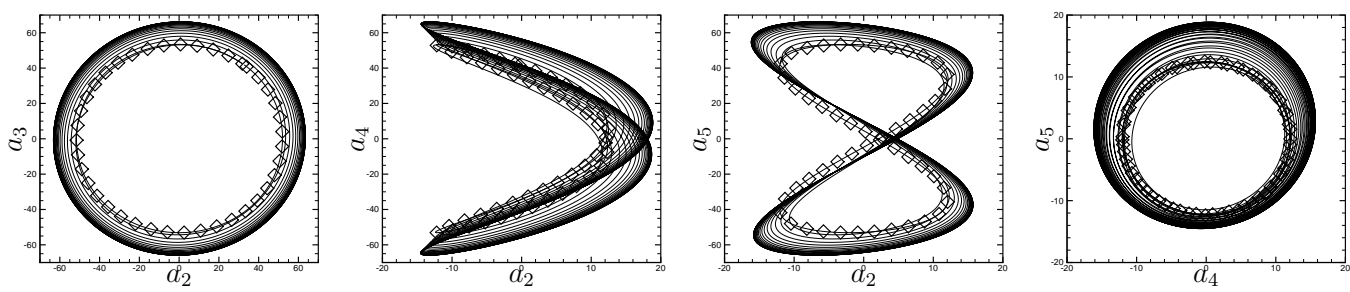

Fig. 9. Comparison of the projected (NS: $\diamond)$ and the predicted (ROM: - ) limit cycles over 1000 vortex shedding periods. 5 modes model.
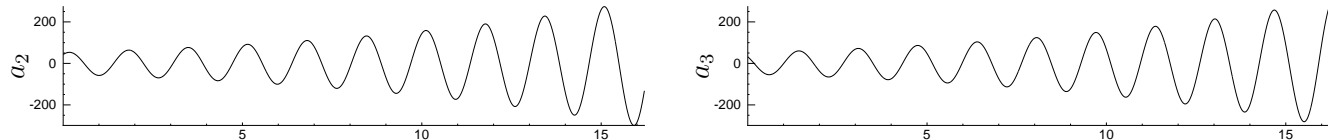

Fig. 10. Temporal evolutions of the predicted POD coefficients over 10 vortex shedding periods. 3 modes model.

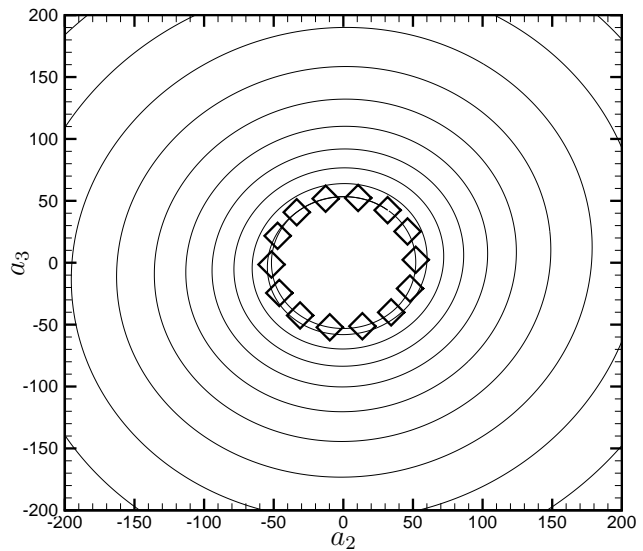

Fig. 11. Comparison of the projected (NS: $\diamond)$ and the predicted (ROM: - ) limit cycles over 1000 vortex shedding periods. 3 modes model.

are sufficient to build a stable ROM. However, in many practical applications (three dimensional flows, turbulent regimes, complex geometries, etc) the number of POD modes that represents $99 \%$ of the total kinetic energy is large. Usually, approximatively $60 \%$ to $80 \%$ of the kinetic energy can be retained, so that reduced order models are unstable. The following section presents methods based on the Navier-Stokes residuals to stabilize reduced order models built with a very low number of modes (namely, 3 or 5 modes in our case).

\section{STABILIZATION OF REDUCED ORDER MODELS}

To overcome errors due to the truncation involved in the POD-Galerkin approach, different kind of POD ROM/Galerin stabilization methods are commonly used. 
The first class of stabilization methods uses eddy viscosity. Since the early works on POD ROM, it was shown that artificial viscosity can help stabilization [23]. A natural way is to add a constant viscosity acting the same way on all POD modes: this is called Heisenberg model [24,25]. The global dimensionless viscosity $1 / R e$ is thus replaced by another one defined as $(1+c) / R e$. The problem is then to determine or to adjust the constant $c>0$ in order to obtain a better accuracy for the POD ROM. Rempfer and Fasel [26] and Rempfer [27] have improved this idea by supposing that the dissipation is not identical on each of the POD modes. Thus, the global viscosity could be replaced by modal viscosities $1 / R e_{i}=\left(1+c_{i}\right) / R e$ on each POD mode $\boldsymbol{\Phi}_{i}$. It is then necessary to determine a set of correction coefficients spanned by $c_{i}$ for $i=1, \ldots, N_{r}$. In [27] it is argued that these eddy viscosities are a function of the coupled modes index $j=1, \ldots, N_{r} / 2$. The coefficients $c_{j}$ are such that $c_{j}=K \times j$ where $K$ is the unique constant to determine or to adjust. More recently, Karniadakis employed a dissipative model called Spectral Vanishing Viscosity Model (SVVM) to formulate alternative stabilization approaches [28] and to improve the accuracy of POD flow models [20]. In this spirit, an optimal spectral viscosity model based on parameters identification technique has been proposed [29].

The second class of stabilization methods consists in calibrating the polynomial coefficients of the POD model [12,30-32]. All the coefficients of tensor $B$ are determined using a least square or an adjoint method so that the predicted coefficients $a_{i}(t)$ are as closed as possible to the eigenvectors of the temporal correlation tensor (see equation (2)). These calibration methods, based on system identification, are very similar to spectral viscosity closures. However, calibration methods allow such a representation of the inter-modal transfers.

The third class of stabilization methods uses a penalty term. This consists in introducing a new term in the reduced order model. Cazemier [33] and Cazemier et al. [34] used modal kinetic equations to determine viscosities to be added on each POD mode. Cazemier [33] supposes that the lack of interaction between the calculated and the non-resolved modes is responsible of a linear divergence of the temporal POD coefficients. To solve this problem another artificial linear coefficient is introduced in the POD dynamical system. The POD ROM writes then:

$$
\frac{\mathrm{d} a_{i}}{\mathrm{~d} t}=\mathcal{A}_{i}+\sum_{j=1}^{N_{r}} \mathcal{B}_{i j} a_{j}+\sum_{j=1}^{N_{r}} \sum_{k=1}^{N_{r}} \mathcal{C}_{i j k} a_{j} a_{k}+\mathcal{H}_{i} a_{i},
$$

where, after some manipulations based on energetic conservation (see [35] for the derivation of the energetic residual),

$$
\mathcal{H}_{i}=-\frac{1}{\lambda_{i}} \sum_{j=1}^{N_{r}} \sum_{k=1}^{N_{r}} \mathcal{C}_{i j k}\left\langle a_{i} a_{j} a_{k}\right\rangle-\mathcal{B}_{i i}
$$


For a compressible flow, Vigo [36] proposed a stabilisation method based on a cubic penalization term in order to prevent a nonlinear amplification. This method seems to give good results but the construction of this cubic term and the resolution of the POD ROM induces high numerical costs. A linear penalty term can also be added to model the pressure term. The drawbacks of all penalization methods is the numerical costs involved in computing the penalization term.

Finally, the last class of stabilization methods that can be found in the literature introduces dissipation directly in the numerical schemes used to build the POD ROM [37]. Instead of using the standard $L^{2}$ inner product in the POD, the Sobolev norm $H^{1}$ is used [38]. However, the level of dissipation has also to be fixed.

The main drawback of all the previous stabilization methods is that there are always a lot of parameters to fix or to optimize. The aim of this section is to derive stabilization methods that involve less empirical parameters.

Let $A^{\left[N_{r}\right]}$ be the model defined by $(21)$ and derived using with $N_{r}$ modes. Unstable models correspond to $N_{r}=3$ or $N_{r}=5$.

The two kinds of stabilization methods presented in what follows use the residual of the Navier-Stokes (NS) operator evaluated with the POD flow fields. These residuals, called POD-NS residuals, are:

$$
\begin{aligned}
& \boldsymbol{R}_{M}(\boldsymbol{x}, t)=\frac{\partial \widetilde{\boldsymbol{u}}}{\partial t}+(\widetilde{\boldsymbol{u}} \cdot \nabla) \widetilde{\boldsymbol{u}}+\nabla \widetilde{p}-\frac{1}{R e} \Delta \widetilde{\boldsymbol{u}}, \\
& R_{C}(\boldsymbol{x}, t)=\boldsymbol{\nabla} \cdot \widetilde{\boldsymbol{u}},
\end{aligned}
$$

where the POD flow fields $\widetilde{u}$ and $\widetilde{p}$ are given by decompositions (11).

We showed that the POD ROM is stable if a sufficient number of modes is taken into account in the model. For instance, model $A^{[11]}$ is stable. Thus, the POD ROM is stable if the POD flow fields get close enough to the exact flow field. The exact flow field writes:

$$
\begin{aligned}
\boldsymbol{u}(\boldsymbol{x}, t) & =\widetilde{\boldsymbol{u}}(\boldsymbol{x}, t)+\boldsymbol{u}^{\prime}(\boldsymbol{x}, t), \\
p(\boldsymbol{x}, t) & =\widetilde{p}(\boldsymbol{x}, t)+p^{\prime}(\boldsymbol{x}, t),
\end{aligned}
$$

where $u^{\prime}$ and $p^{\prime}$ denote the fine scales that are not resolved by the POD ROM. Unfortunately, the exact resolution of the fine scales equations (with solutions $\boldsymbol{u}^{\prime}$ and $p^{\prime}$ ) requires computational costs similar to those required for solving the complete Navier-Stokes equations. The objective of the following section is thus to derive stabilization methods that make use of approximations of these fine scales. 


\subsection{Residuals based stabilization method: $\operatorname{model} B^{\left[N_{r} ; K\right]}$}

The goal of this method is to approximate the fine scales $u^{\prime}$ and $p^{\prime}$ onto some adapted basis functions. The exact flow field is hence approximated as:

$$
\begin{aligned}
& \boldsymbol{u}(\boldsymbol{x}, t)=\underbrace{\sum_{i=1}^{N_{r}} a_{i}(t) \boldsymbol{\phi}_{i}(\boldsymbol{x})}_{\widetilde{\boldsymbol{u}}(\boldsymbol{x}, t)}+\underbrace{\sum_{i=N_{r}+1}^{N_{r}+K} a_{i}(t) \boldsymbol{\phi}_{i}^{\prime}(\boldsymbol{x})}_{\boldsymbol{u}^{\prime}(\boldsymbol{x}, t)}, \\
& p(\boldsymbol{x}, t)=\underbrace{\sum_{i=1}^{N_{r}} a_{i}(t) \psi_{i}(\boldsymbol{x})}_{\widetilde{p}(\boldsymbol{x}, t)}+\underbrace{\sum_{i=N_{r}+1}^{N_{r}+K} a_{i}(t) \psi_{i}^{\prime}(\boldsymbol{x})}_{p^{\prime}(\boldsymbol{x}, t)} .
\end{aligned}
$$

If the basis functions $\phi_{i}^{\prime}$ and $\psi_{i}^{\prime}$ are respectively equal to $\phi_{i}$ and $\psi_{i}$ the energetic representation is improved. Indeed, the more POD modes are retained, the better is the energetic representation. This is a classical remark of POD. However, it is not granted that POD modes are optimal to stabilize the ROM. If we suppose that a sufficient amount of energy is captured with $N_{r}$ modes, it is not necessary to add any more energy in the POD ROM, but rather some viscous dissipation. For instance, model $A^{[5]}$, i.e. model $B^{[3 ; 2]}$ with $\boldsymbol{\Phi}^{\prime} \equiv \boldsymbol{\Phi}$ in (24), is not stable. However, it will be demonstrated that another 5-modes model with $N_{r}=3$ and $K=2$ is stable using different modes $\boldsymbol{\Phi}^{\prime}$. It is well known that the residuals of the governing equations play a major role to stabilize dynamical systems [14]. The leading idea of this section is thus to take $\phi_{i}^{\prime}$ and $\psi_{i}^{\prime}$ as being the POD basis functions of the POD-NS residuals defined by (22). The method is the following.

\section{Algorithm 1 (Residuals based stabilization)}

(1) Integrate the $R O M A^{\left[N_{r}\right]}$ to obtain $a_{i}(t)$ and compute $N_{s}$ coefficients $a_{i}\left(t_{k}\right), k=1, \ldots, N_{s}$.

(2) Compute the POD flow fields $\widetilde{\boldsymbol{u}}\left(\boldsymbol{x}, t_{k}\right)=\sum_{i=1}^{N_{r}} a_{i}\left(t_{k}\right) \boldsymbol{\phi}_{i}(\boldsymbol{x}), \widetilde{p}\left(\boldsymbol{x}, t_{k}\right)=$ $\sum_{i=1}^{N_{r}} a_{i}\left(t_{k}\right) \psi_{i}(\boldsymbol{x})$, and then the POD-NS residuals $\boldsymbol{R}_{M}\left(\boldsymbol{x}, t_{k}\right)$ and $R_{C}\left(\boldsymbol{x}, t_{k}\right)$.

(3) Compute the POD modes $\boldsymbol{\phi}_{i}^{\prime}(\boldsymbol{x})$ and $\psi_{i}^{\prime}(\boldsymbol{x})$ of the POD-NS residuals $\boldsymbol{R}_{M}\left(\boldsymbol{x}, t_{k}\right)$ and $R_{C}\left(\boldsymbol{x}, t_{k}\right)$.

(4) Add the $K$ first residual modes $\phi_{i}^{\prime}$ and $\psi_{i}^{\prime}$ to the existing POD basis $\phi_{i}$ and $\psi_{i}$ (using Gram-Schmidt process) and built a new ROM (here the mass and flow rate constraints are important).

The reduced order model obtained with this algorithm is noted $B^{\left[N_{r} ; K\right]}$ where $N_{r}$ is the number of initial POD basis functions and $K$ is the number of POD 
residuals modes. Results of this model are presented in section 4.3.

\subsection{SUPG and VMS methods: models $C^{\left[N_{r}\right]}$ and $D^{\left[N_{r}\right]}$}

The Streamline Upwind Petrov-Galerkin (SUPG) and the Variational Multiscale (VMS) methods are devised to provide appropriate modeling and stabilizations for the numerical solution of the Navier-Stokes equations. The SUPG method is a simplified version of the complete VMS method, and the main steps leading to these models are described in [14].

The main idea of both SUPG and VMS methods is to approximate the fine scales by:

$$
\begin{aligned}
& \boldsymbol{u}^{\prime} \simeq-\tau_{M} \boldsymbol{R}_{M} \\
& p^{\prime} \simeq-\tau_{C} R_{C},
\end{aligned}
$$

where $\tau_{M}$ and $\tau_{c}$ denote some constants to be fixed.

The SUPG and VMS reduced order models can be formally written:

$$
\begin{aligned}
\sum_{j=1}^{N_{r}}\left(L_{i j}^{(m)}+\beta L_{i j}^{(r)}\right) \frac{\mathrm{d} a_{j}}{\mathrm{~d} t} & =\sum_{j=1}^{N_{r}}\left(B_{i j}^{(m)}+\alpha B_{i j}^{(c)}\right) a_{j}+\sum_{j=1}^{N_{r}} \sum_{k=1}^{N_{r}} C_{i j k}^{(m)} a_{j} a_{k} \\
& +F_{i}(t)
\end{aligned}
$$

where the "penalization" term $F_{i}(t)$ is defined as follows:

- For the SUPG reduced order model, noted $C^{\left[N_{r}\right]}$, we have:

$$
F_{i}^{S U P G}(t)=\left(\widetilde{\boldsymbol{u}} \cdot \boldsymbol{\nabla} \boldsymbol{\phi}_{i}+\nabla \psi_{i}, \tau_{M} \boldsymbol{R}_{M}(\boldsymbol{x}, t)\right)_{\Omega}+\left(\boldsymbol{\nabla} \cdot \boldsymbol{\phi}_{i}, \tau_{C} R_{C}(\boldsymbol{x}, t)\right)_{\Omega} .
$$

- For the VMS reduced order model, noted $D^{\left[N_{r}\right]}$, we have:

$$
\begin{aligned}
F_{i}^{V M S}(t)=F_{i}^{S U P G}(t) & +\left(\widetilde{\boldsymbol{u}} \cdot\left(\boldsymbol{\nabla} \boldsymbol{\phi}_{i}\right)^{T}, \tau_{M} \boldsymbol{R}_{M}(\boldsymbol{x}, t)\right)_{\Omega} \\
& -\left(\boldsymbol{\nabla} \boldsymbol{\phi}_{i}, \tau_{M} \boldsymbol{R}_{M}(\boldsymbol{x}, t) \otimes \tau_{M} \boldsymbol{R}_{M}(\boldsymbol{x}, t)\right)_{\Omega}
\end{aligned}
$$

The parameters $\tau_{M}$ and $\tau_{C}$ can be found using some scaling arguments (see [39] for more details), so that no modelisation is required. This can lead to an universal POD ROM closure model. However, in what follows, parameters $\tau_{M}$ and $\tau_{C}$ are determined using an optimal formulation, so that the temporal coefficients $a_{i}(t)$ fit as best as possible the eigenvectors of the correlations tensor (3).

For clarity reasons, table 1 summarizes the different ROM introduced above, where $N_{r}$ denotes the number of POD modes used to compute the ROMs. 
Table 1

\begin{tabular}{|c|c|}
\hline ROM & Method \\
\hline$A^{\left[N_{r}\right]}$ & No stabilization \\
$B^{\left[N_{r}, K\right]}$ & Stabilization with $K$ residuals POD modes \\
$C^{\left[N_{r}\right]}$ & SUPG stabilization \\
$D^{\left[N_{r}\right]}$ & VMS stabilization \\
\hline
\end{tabular}

Brief description of the different ROMs. $N_{r}$ denote the number of the retained POD modes.

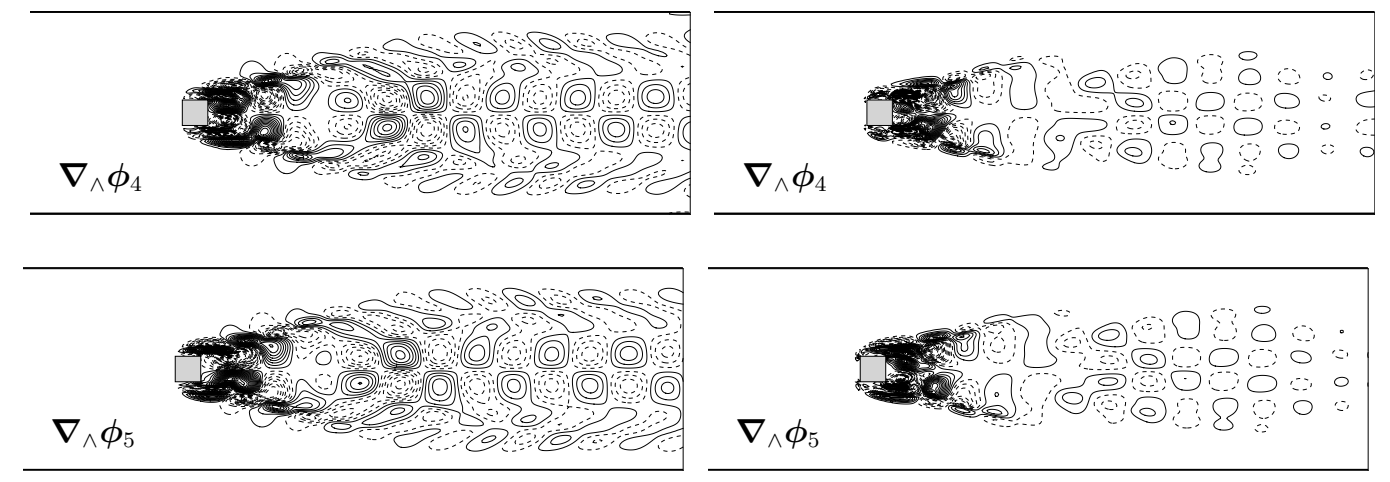

Fig. 12. Comparison between original POD modes (left, model $A^{[5]}$ ) and residuals modes (right, model $B^{[3 ; 2]}$ ). Dashed lines represent negative values.

\subsection{Results of stabilization methods}

In this section we take exactly the same configuration and parameters than those used in §3.2. The confined square cylinder wake flow for $R e=200$ and the ROMs are built using $N_{s}=80$ snapshots uniformly distributed over one vortex shedding period. The model based on residual modes, $B^{\left[N_{r} ; K\right]}$, is integrated following $§ 4.1$. The SUPG model, $C^{\left[N_{r}\right]}$, and the VMS model, $D^{\left[N_{r}\right]}$, are integrated using (27) and (28) respectively. The unstable configurations observed in $\S 3.2$ are studied, i.e. $N_{r}=5$ and $N_{r}=3$. Moreover, for model $B^{\left[N_{r} ; K\right]}$, only $K=2$ residual modes are used. These two additional modes present a different behavior than POD modes 4 and 5 used to build model $A^{[5]}$ (see figure 12 ).

The temporal evolutions of the predicted POD coefficients $a_{i}(t)$ obtained with models $B, C$ and $D$, over 40 vortex shedding periods, are presented in figures 13 and 15 for $N_{r}=5$ and $N_{r}=3$ respectively. The three reduced order models provide an accurate description of the asymptotic attractor (compare with results in figure 6), and results are indistinguishable between model $B$, $C$ and $D$. The limit cycles obtained with models $B, C$ and $D$ are represented in figures 14 and 16 for $N_{r}=5$ and $N_{r}=3$ respectively. These limit cycles 

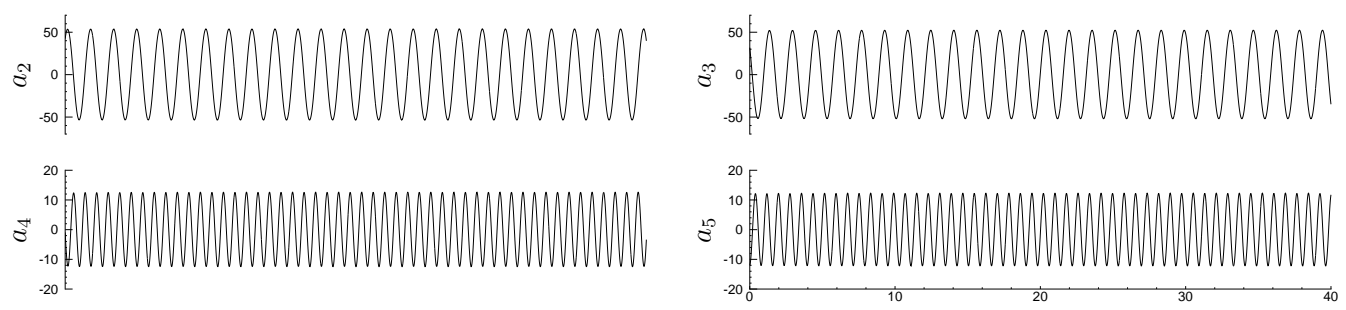

Fig. 13. Temporal evolutions of the predicted POD coefficients over 40 vortex shedding periods. 5 modes model with stabilization. (Indistinguishable difference between models $B^{[5,2]}, C^{[5]}$ and $D^{[5]}$.)
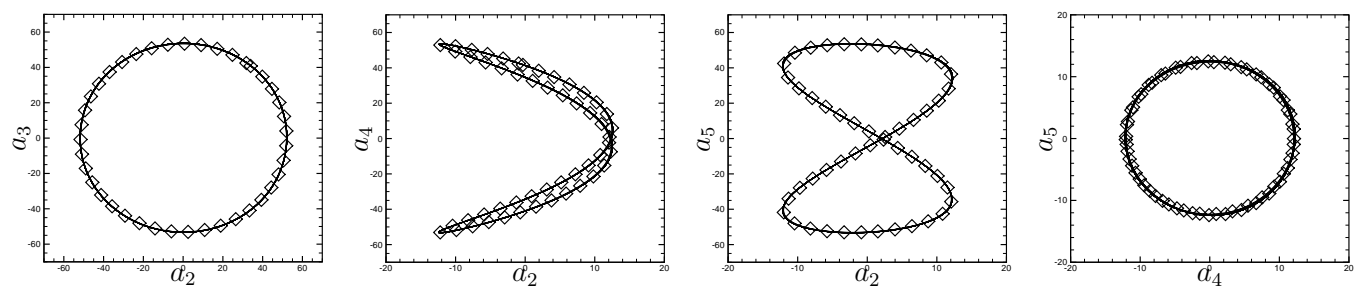

Fig. 14. Comparison of the projected (NS: $\diamond)$ and the predicted (ROM: - ) limit cycles over 1000 vortex shedding periods. 5 modes model with stabilization. (Indistinguishable difference between models $B^{[5,2]}, C^{[5]}$ and $D^{[5]}$.)
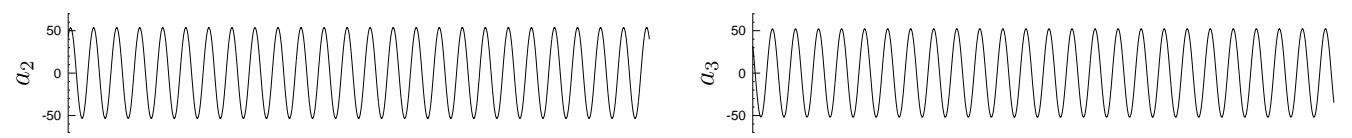

Fig. 15. Temporal evolutions of the predicted POD coefficients over 40 vortex shedding periods. 3 modes model with stabilization. (Indistinguishable difference between models $B^{[3,2]}, C^{[3]}$ and $D^{[3]}$.)

represent 1000 vortex shedding periods. There are no difference between results obtained from the three models. These limit cycles are compared to exact ones obtained by DNS (projection of the snapshots onto the POD basis) in the figures. Excellent agreements are observed between all these limit cycles, thus validating all the stabilization methods described in $\S 4.1$ and $\S 4.2$.

In order to highlight the differences between the stabilization methods presented above, we study the $L^{2}$ norm of the POD-NS residuals introduced in (22). Figures 17(a) and 18(a) show the temporal evolutions of the $L_{2}$ norms of the POD-NS residuals obtained initial model $A$ (obtained from (21) or (26) with $F_{i}=0$ ) with $N_{r}=5$ and $N_{r}=3$ respectively. For sake of clarity, only 20 vortex shedding periods are represented. An initial growth and then an asymptotic limit is reached with $N_{r}=5$. This can be explained by the fact that the dynamic converges towards another attractor (see figure 7). On the contrary, an exponential divergence occurs with $N_{r}=3$ (see also the divergence in figure 11). We compare the effectiveness of models $B, C$ and $D$ in figures 17(b) and 18(b). All stabilized reduced order models are accurate (low 


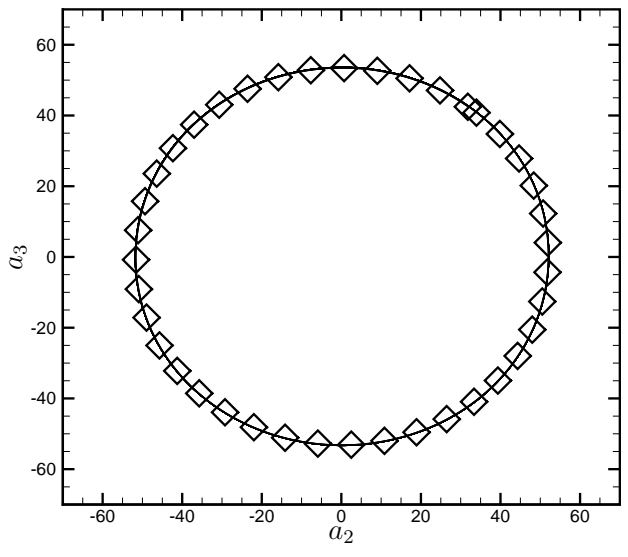

Fig. 16. Comparison of the projected (NS: $\diamond)$ and the predicted (ROM: - ) limit cycles over 1000 vortex shedding periods. 3 modes model with stabilization. (Indistinguishable difference between models $B^{[3,2]}, C^{[3]}$ and $D^{[3]}$.)

values of the POD-NS residuals norm). For the $N_{r}=5$ or $N_{r}=3$, models $C$ and $D$ are more accurate than model $B$ (lower residuals). Moreover, the VMS model, $D$, is better than the SUPG one, $C$, but without significant differences between them. In our reduced order modeling the SUPG method seems to be sufficient to obtain an accurate POD ROM. However, the numerical costs required for both SUPG and VMS methods are similar. To conclude on the

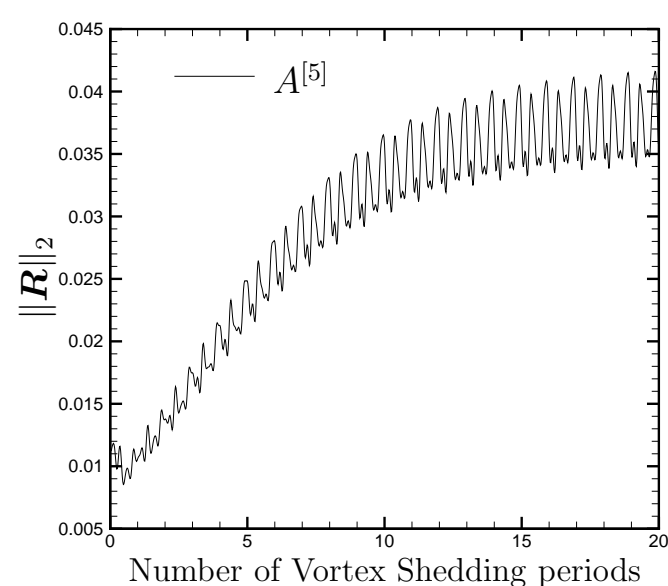

(a) Initial model.

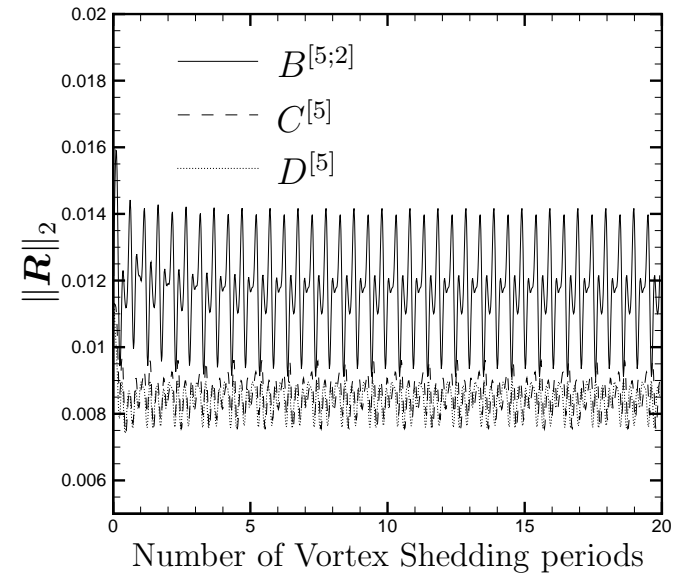

(b) Stabilized models.

Fig. 17. Temporal evolution of the $L^{2}$ norm of the Navier-Stokes residuals computed using a 5-modes ROM.

first stabilization method, it is noticeable that although models $B^{[3 ; 2]}$ and $A^{[5]}$ used 5 modes, only model $B^{[3 ; 2]}$ is stable. It is thus not necessary to include a lot of modes in the POD basis in order to obtain a stable model, but only to add some appropriate "damping" modes (see figure 12). 


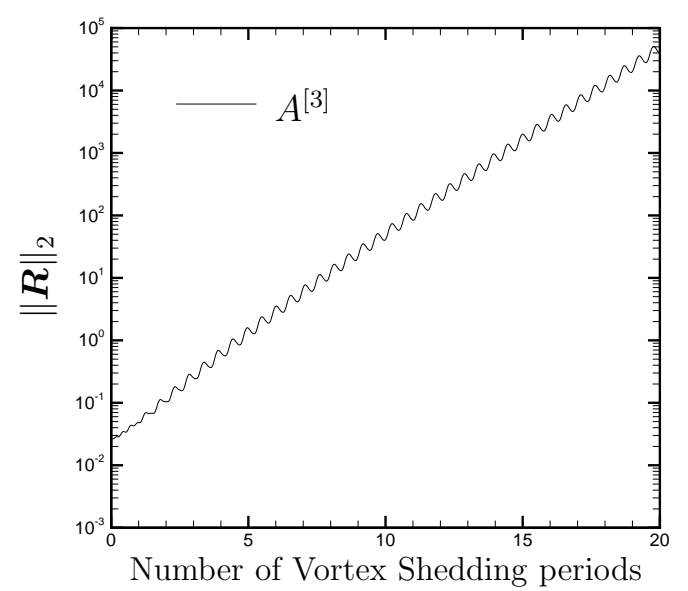

(a) Initial model.

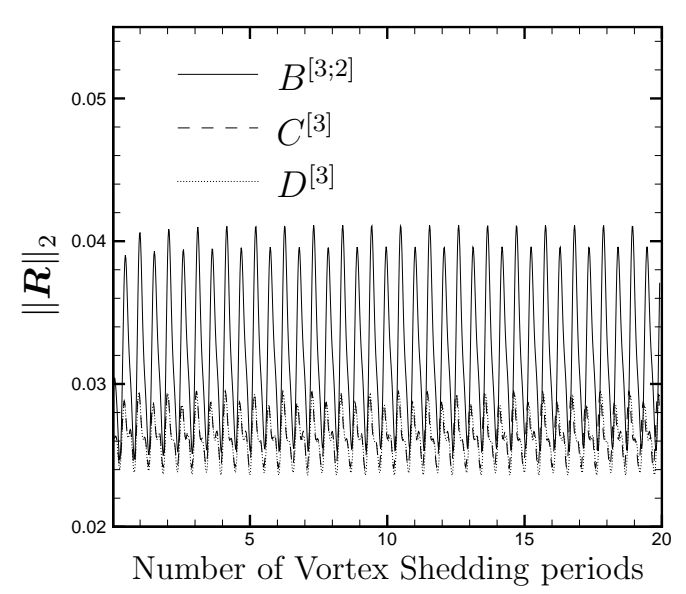

(b) Stabilized models.

Fig. 18. Temporal evolution of the $L^{2}$ norm of the Navier-Stokes residuals computed using a 3-modes ROM.

\section{IMPROVEMENT OF THE FUNCTIONAL SUBSPACE}

Since the POD was first introduced in turbulence by Lumley [18] in 1967 as an unbiased definition of the coherent structures in a turbulent flow, POD was used to analyze physical characteristics of turbulent flows. More recently, Reduced Order Models based on POD are found as being an efficient tool for flow control purpose (see [40-42,29,17] for examples). Indeed, the use of POD ROM allows to reduce significantly the CPU time during numerical simulation and also to reduce the memory storage, an essential feature when adjoint based optimal control methods are used. Different optimization methods that couple POD ROM and optimal control have been taken under consideration. The main drawback for flow control is that the POD basis is only able to give an optimal representation of the snapshots set from which it was derived. The approximation properties of the basis can be greatly degraded under variation of some input system parameters values, as control parameters [15-17]. For flow control purposes, some special care has to be taken to build the POD basis functions. One solution is to use an a priori global database composed by several dynamics. For example, it is possible to use a database composed by snapshots that correspond to different control laws [17] or different Reynolds numbers [43]. One efficient way to do that is either by Centroidal Voronoi Tessellations (CVT) [44] or by using an ad-hoc time-dependent control law that generates a flow representing a large band of dynamics $[45,42,29]$. We privilege the idea of updating the POD basis during the simulation. Trust Region Proper Orthogonal Decomposition originally introduced by Fahl [46] is an example of such ideas (see Refs. $[47,17]$ ). The main drawback of the TRPOD method is that the POD basis has to be re-actualized. Each actualization requires a large computational effort since it involves DNS. 

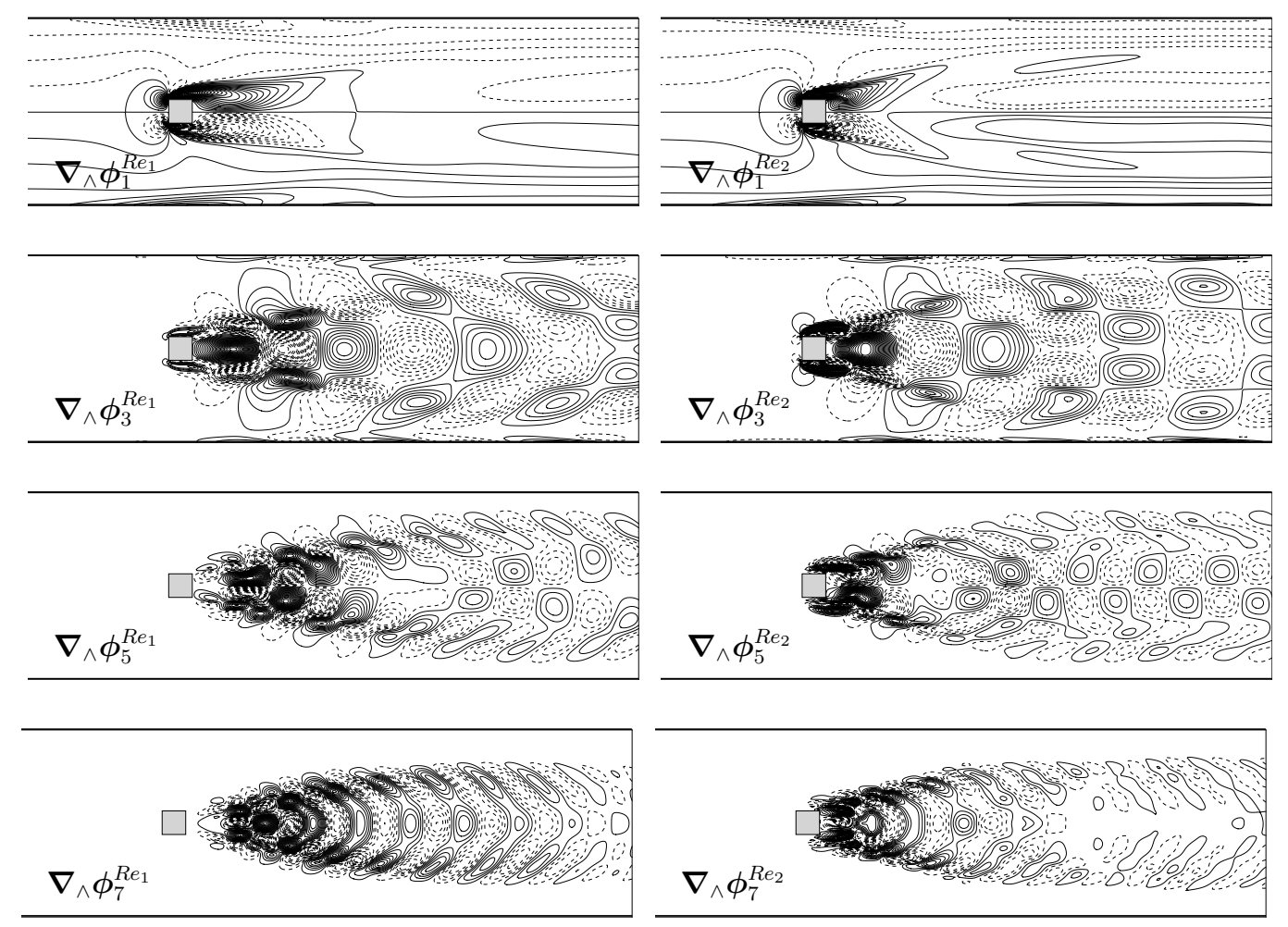

Fig. 19. Comparison of the iso-vorticity representation of some POD modes. The initial POD basis at $R e_{1}=100$ (left) and the target POD basis at $R e_{2}=200$ (right). Dashed lines represent negative values.

The aim of this section is thus to present efficient methods to improve - or actualize - the functional subspace. As it was mentioned, the underlying idea is to adapt the basis when input system parameters change (Reynolds number, control parameters, etc). For simplicity reasons, we will only focus on Reynolds number modifications, but the forthcoming process is easily transposable to other parameters modifications. Here, for instance, our goal is to obtain the target basis built at $R e_{2}=200$ starting from the initial basis built at $R e_{1}=$ 100. These two basis are quite different, especially for the mean flow mode $\phi_{1}$ (see figure 19).

In what follows two methods are considered.

- The objective of the first method is to improve the basis using residuals based approximations of the missing fields $\boldsymbol{u}^{\prime}$ and $p^{\prime}$. This is exactly the same idea that was used in (§4.1), applied iteratively (§5.1).

- The aim of the second method is to modify the POD database by means of DNS simulations ( $(5.2)$.

These two methods are based on an iterative process. The current POD basis obtained during the actualization process is simply denoted by $\boldsymbol{\Phi} \equiv \boldsymbol{\Phi}^{(n)}$ where $n$ is the number of iterations considered (number of POD basis actualization 
cycles). Obviously, $\boldsymbol{\Phi}^{(0)}=\boldsymbol{\Phi}^{R e_{1}}$, and we expect at least that $\boldsymbol{\Phi}^{(+\infty)}=\boldsymbol{\Phi}^{R e_{2}}$. The convergence criterion tested in this study is the best projection of $\boldsymbol{\Phi}^{(n)}$ onto $\boldsymbol{\Phi}^{R e_{2}}$, noted $\boldsymbol{\Phi} \cdot \boldsymbol{\Phi}^{R e_{2}}$. For our Navier-Stokes test case with $R e_{1}=100$ and $R e_{2}=200$, the initial value of the convergence criterion is $\boldsymbol{\Phi}^{R e_{1}} \cdot \boldsymbol{\Phi}^{R e_{2}} \approx 0.5$. The expected final value is naturally $\boldsymbol{\Phi}^{(n)} \cdot \boldsymbol{\Phi}^{R e_{2}} \approx 1$, with the smallest possible number of iterations $n$.

\subsection{A Krylov-like method}

The first method used to improve the functional subspace uses successive application of the Navier-Stokes operator on the residuals. This method is nothing but an iterative version of the stabilization process introduced in \$4.1. We have seen that this method does a good job to stabilize POD ROM, so it is reasonable to investigate its performance to improve the functional subspace. This subspace adaptation is described by the following algorithm and is schematically represented in figure 20. The numerical integration of the ROM (21) is always performed at the target Reynolds number $R e \equiv R e_{2}$ (the Reynolds number $R e$ is required to evaluate $B^{(m)}$, see equation (16b)). Otherwise, all the POD coefficients, i.e. $L^{(m)}, L^{(r)}, B^{(m)}, B^{(c)}, C^{(m)}$, are built using the current updated POD basis, $\boldsymbol{\Phi}=\boldsymbol{\Phi}^{(n)}$, following (16).

\section{Algorithm 2 (Krylov like adaptation method)}

Start with the POD basis to be improved, $\boldsymbol{\Phi}_{i}$ with $i=1, \ldots, N_{r}$ (in our case built for $\left.R e=R e_{1}\right)$. Let $N_{0}=N_{r}$ and $\mathcal{T}=[0, T]$ be an observation period.

(1) Build and solve the corresponding $R O M$ over $\mathcal{T}$ with $R e=R e_{2}$ to obtain $a_{i}(t)$ and extract $N_{s}$ snapshots $a_{i}\left(t_{k}\right)$ with $i=1, \ldots, N_{r}$ and $k=$ $1, \ldots, N_{s}$. Compute the flow fields $\left.\widetilde{\boldsymbol{u}}\left(\boldsymbol{x}, t_{k}\right)\right), \widetilde{p}\left(\boldsymbol{x}, t_{k}\right)$ from (11).

(2) Compute the POD-NS residuals $\boldsymbol{R}\left(\boldsymbol{x}, t_{k}\right)=\left(\boldsymbol{R}_{M}\left(\boldsymbol{x}, t_{k}\right), R_{C}\left(\boldsymbol{x}, t_{k}\right)\right)^{T}$ from (22).

(3) Compute the POD modes $\widetilde{\boldsymbol{\Phi}}(\boldsymbol{x})=(\widetilde{\boldsymbol{\phi}}(\boldsymbol{x}), \widetilde{\psi}(\boldsymbol{x}))^{T}$ from the POD-NS residuals database $\boldsymbol{R}\left(\boldsymbol{x}, t_{k}\right), k=1, \ldots, N_{s}$.

(4) Add the $K$ firsts residual modes $\widetilde{\boldsymbol{\Phi}}(\boldsymbol{x})$ to the existing POD basis $\boldsymbol{\Phi}_{i}(\boldsymbol{x})$ (using Gram-Schmidt process)

- $\boldsymbol{\Phi} \leftarrow \boldsymbol{\Phi}+\boldsymbol{\Psi}$

- $N_{r} \leftarrow N_{r}+K$

- If $N_{r}$ is below than a threshold, $N_{\max }$, return to 1 . Else, go to 5 .

(5) Do step (1). From fields $\widetilde{\boldsymbol{u}}$ and $\widetilde{p}$, perform a new POD compression from with $N_{r}=N_{0}$.

- If a convergence criterion is satisfied, stop. Else, return to 1. 


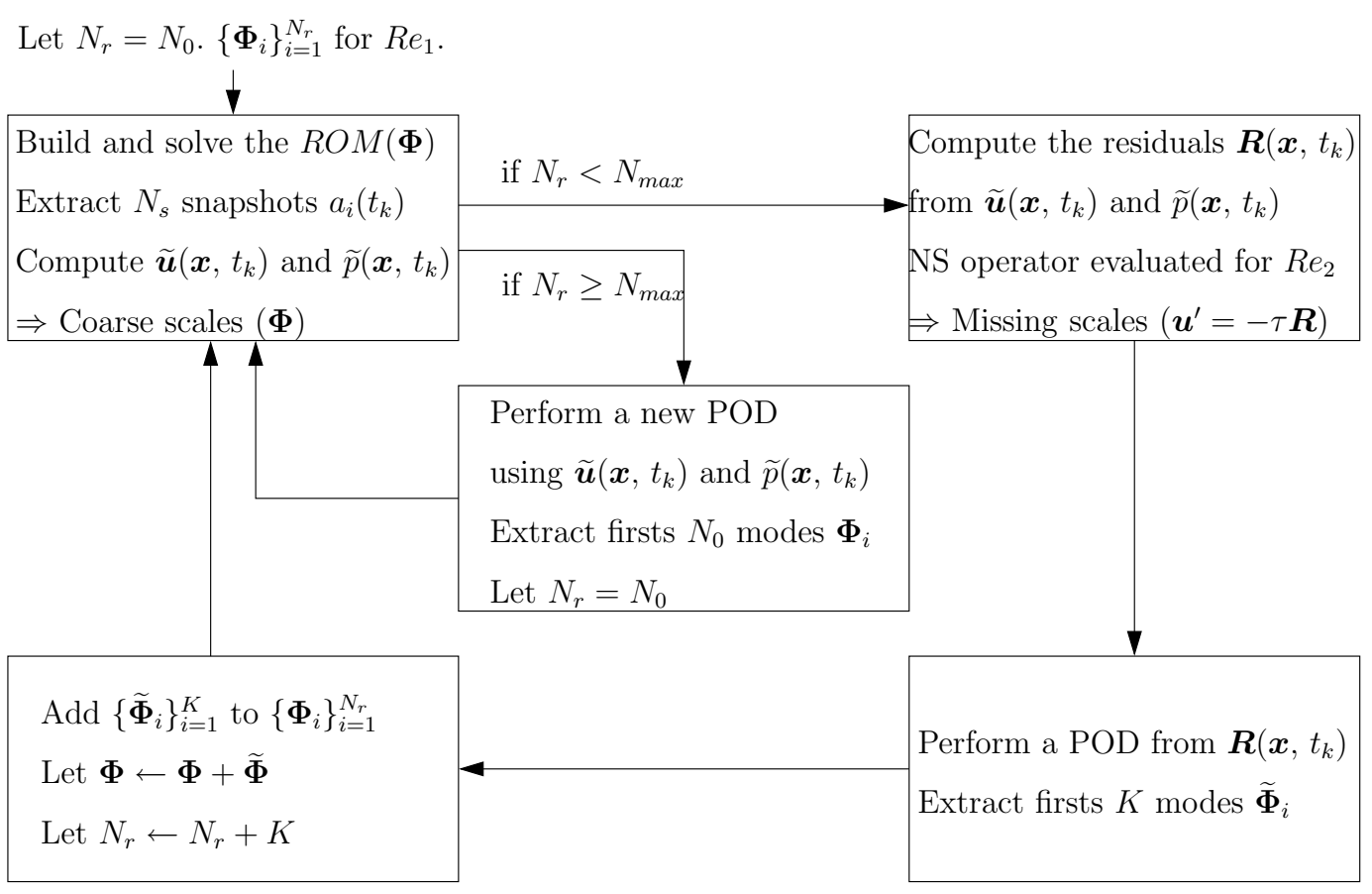

Fig. 20. Schematic representation of the functional adaptation process based on a Krylov-like method.

This algorithm is a simplified version of a generalized minimal residual (GMRES) algorithm for linear system (see [48] for more details about GMRES method).

Before testing this adaptation method on the two-dimensional confined square cylinder wake flow governed by the Navier-Stokes equations ( $\$ 5.1 .2)$, a simple one dimensional test case is performed on the Burgers equation (§5.1.1).

\subsubsection{A one dimensional test case: the Burgers equation}

The Burgers equation, in its dimensionless form, writes:

$$
\mathcal{L}_{B}(u)=\frac{\partial u}{\partial t}+\frac{1}{2} \frac{\partial u^{2}}{\partial x}-\frac{1}{R e} \frac{\partial^{2} u}{\partial x^{2}}=0
$$

with initial condition ${ }^{6}$

$$
u(x, 0)=\sin \left(\pi \frac{\tan \left(c_{s}(2 x-1)\right)}{\tan \left(c_{s}\right)}\right) \quad \text { and } \quad c_{s}=1.3
$$

and boundary conditions

$$
\begin{aligned}
& u(0, t)=0, \\
& u(L, t)=0 .
\end{aligned}
$$

$\overline{6}$ This value of $c_{s}$ is chosen to obtain a shock wave in the domain $\mathcal{D}$. 

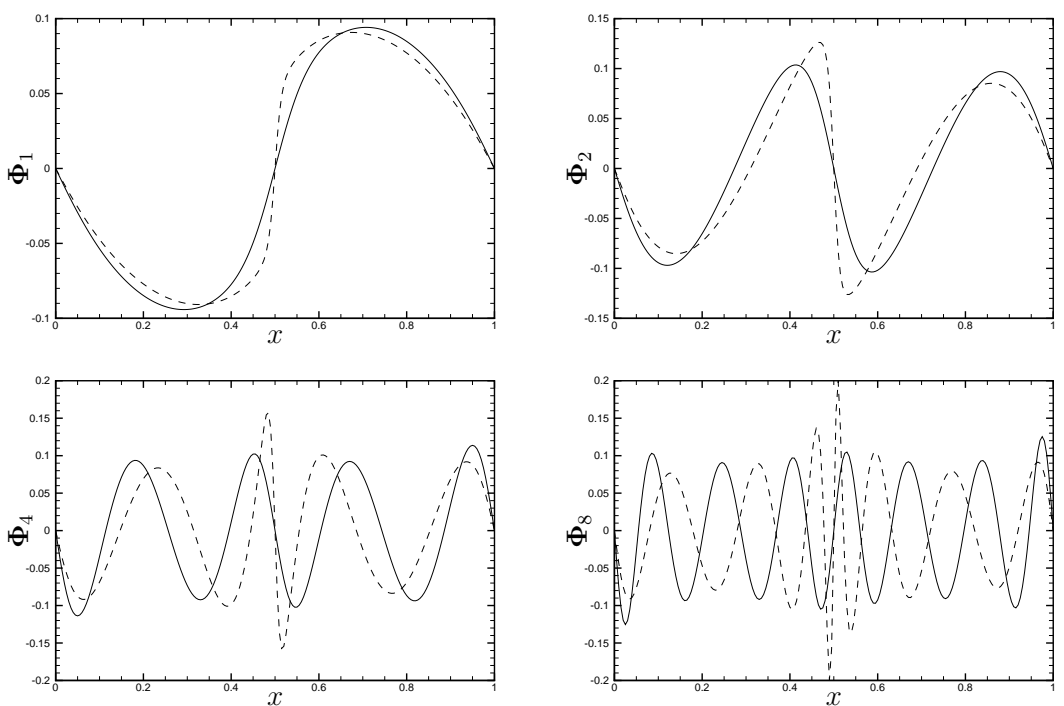

Fig. 21. Comparison of few POD basis functions obtained for $R e_{1}=50(-)$ and $R e_{2}=300(---)$.

This equation is solved onto the domain $\mathcal{D}$ defined by:

$$
\mathcal{D}=\{(x, t) \in[0,1] \times[0,1]\}
$$

We chose the initial and target POD basis, $\boldsymbol{\Phi}^{R e_{1}}$ and $\boldsymbol{\Phi}^{R e_{2}}$, such that $\boldsymbol{\Phi}^{R e_{1}}$. $\Phi^{R e_{2}} \approx 0.5$, i.e. $R e_{1}=50$ and $R e_{2}=300$ in this context. We used 40 snapshots uniformly distributed over the whole observation domain $\mathcal{T}=[0,1]$. Figure 21 shows a comparison between few POD basis functions obtained for $R e_{1}$ and $R e_{2}$. Significant differences are observable. Results of the adaptation process are presented in figure 22 which shows the evolution of the convergence criterion versus the number of iterations. Convergence is obtained in less than 6 iterations. In other words, the POD basis at $R e_{2}$ is obtained from that at $R e_{1}$ using only 6 integration of the POD ROM, without any DNS. The POD basis functions at $R e_{2}$ can even be determined starting from one mode (the normalized intial condition), or from any given basis. This represents a very efficient method to actualize a POD basis for the 1D Burgers equation. It is then of interest to see if this adaptation method can provide as good results for the Navier-Stokes equations.

\subsubsection{The confined square cylinder wake flow}

For the confined square cylinder wake flow (2D Navier-Stokes equations), we take $R e_{1}=100, R e_{2}=200$ and we also use 40 snapshots distributed uniformly over one vortex shedding period $T$, so that $\mathcal{T}=[0, T]$, of the $2 \mathrm{D}$ confined square cylinder wake flow. During the adaptation process, the vortex shedding period $T$ has to be actualized in consequence. Results are presented in figure 23. Unfortunately, no convergence is obtained. The algorithm is stopped 


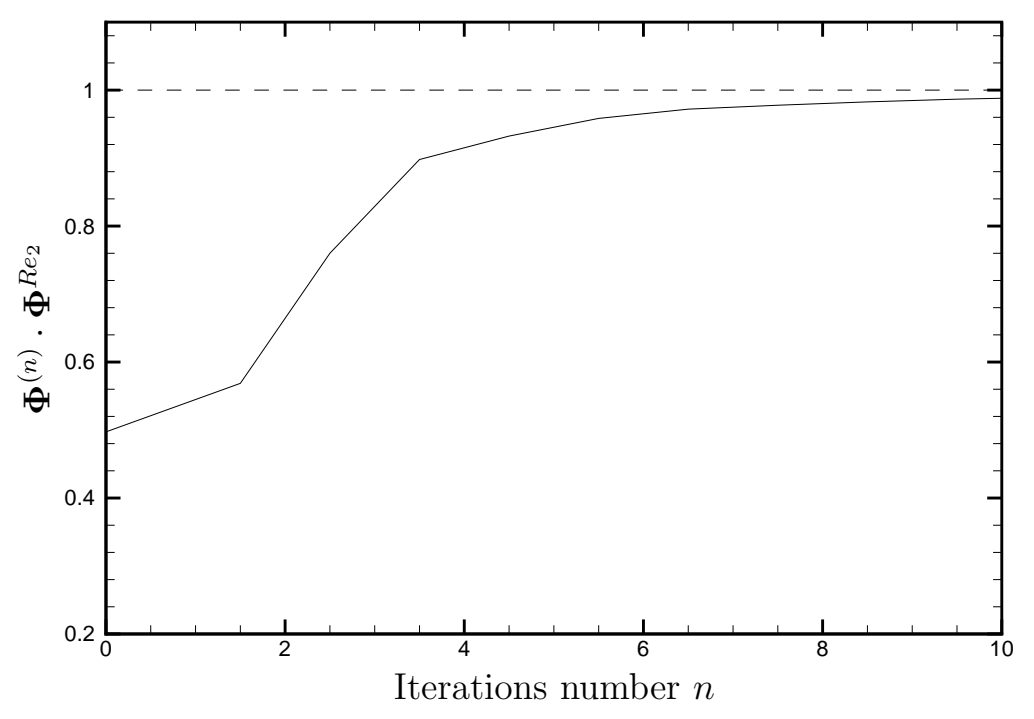

Fig. 22. Evolution of the convergence criterion versus the number of iterations for the $1 \mathrm{D}$ burgers equation.

when the computational costs were estimated to be larger than those necessary using only DNS. Same results are obtained using 400 snapshots uniformly distributed over an observation period $\mathcal{T}=[0,10 T]$, not presented here. The information contained into the Navier-Stokes residuals are not sufficient to improve the POD basis functions for a dynamical change, but they are sufficient to stabilized the ROM for a given dynamic (see $\S 4.1$ ). One possible explanation is that the approximation of the missing scales, $\boldsymbol{u}^{\prime}(\boldsymbol{x}, t)=-\tau_{M} \boldsymbol{R}_{M}(\boldsymbol{x}, t)$ and $p^{\prime}(\boldsymbol{x}, t)=-\tau_{C} R_{C}(\boldsymbol{x}, t)$, is only valid for fine scales, i.e. the ones that are not represented due to the truncation of the POD basis. Residual modes have just dissipative behavior. We can ask if it is possible to find good values for parameters $\tau_{M}$ and $\tau_{C}$ for "quite large" missing scale. The answer is not so clear yet. One solution could be to look for $\boldsymbol{U}^{\prime}(\boldsymbol{x}, t)=M(t) \boldsymbol{R}(\boldsymbol{x}, t)$, where $M \in \mathbb{R}^{3 \times 3}$ for the $2 \mathrm{D}$ Navier-Stokes equations.

A step toward the full GMRES algorithm was also made. Few Arnoldi modes, $\boldsymbol{\Phi}_{n}^{\prime \prime}=A \boldsymbol{\Phi}_{n}^{\prime}, \boldsymbol{\Phi}_{n}^{\prime \prime \prime}=A \boldsymbol{\Phi}_{n}^{\prime \prime}, \ldots$, have been added to the initial basis. The operator $A$ denotes the linear operator obtained after an adapted discretization of the Navier-Stokes equations, so that $A \boldsymbol{U}=b(\boldsymbol{U})$. The results have not been really improved. A large Arnoldi basis should be certainly necessary, but the numerical costs required to generate (and to solve) the POD ROM become prohibitive.

The following section presents another kind of algorithm that couples POD ROM with DNS. If the Navier-Stokes solution lives on the same attractor (no dynamic change), the DNS can be greatly accelerated using POD basis functions. Indeed, a Galerkin free reduced order model is recently used as DNS accelerator [49] . 


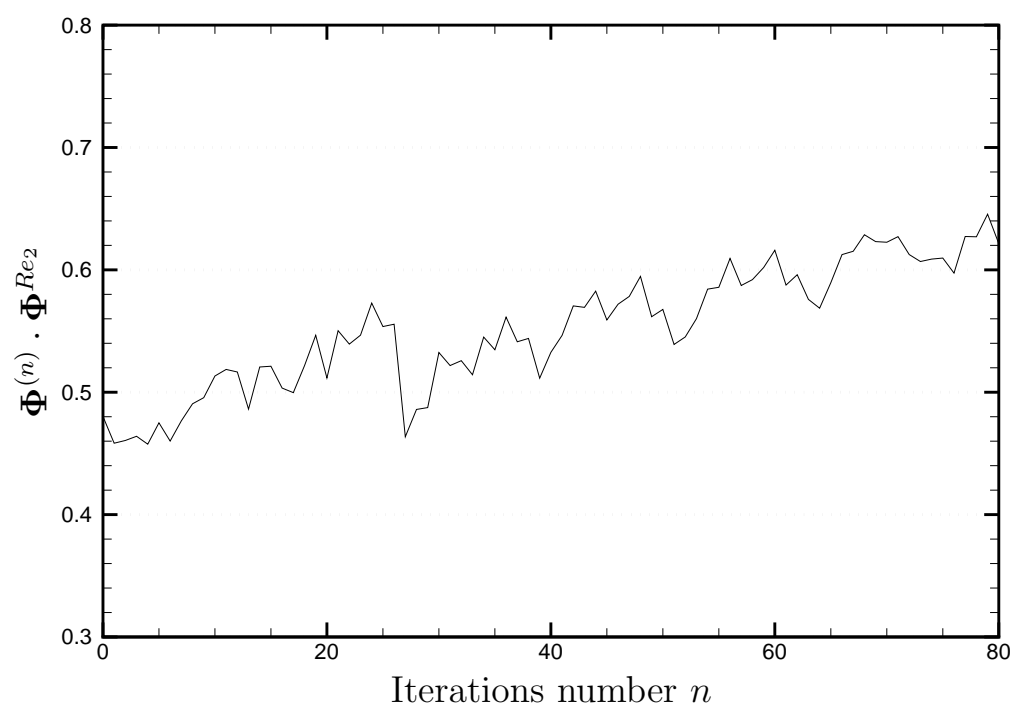

Fig. 23. Evolution of the convergence criterion versus the number of iterations for the 2D Navier-Stokes equations.

$\longmapsto$ ROM $\frac{\text { DNS }}{\boldsymbol{U}\left(\boldsymbol{x}, t_{k-1}\right)}$ ROM $\frac{\text { DNS }}{\boldsymbol{U}\left(\boldsymbol{x}, t_{k}\right)}$ ROM $\frac{\text { DNS }}{\boldsymbol{U}\left(\boldsymbol{x}, t_{k+1}\right)}$ Time

Fig. 24. Schematic representation of the hybrid method DNS-POD ROM to improve the POD subspace.

\subsection{An hybrid DNS/POD ROM method}

It has been demonstrated that the percentage of the reconstruction energy decreases rapidly outside the temporal interval defined by the snapshots database for three dimentional flows [30]. Thus, the aim of the present methodology is to update the database statistics when time evolves. By doing this, the POD basis is actualized and represents with high fidelity the current flow. The main idea is to implement a process allowing to replace older snapshots with new ones at low numerical costs. These new snapshots can be obtained using few DNS iterations. Once a new POD basis is available, a new ROM is constructed and integrated until a new snapshot is needed. Then, the process is repeated. We chose to take snapshot periodically. A schematic representation of the algorithm is presented in figure 24. Let us denote $R e_{1}$ the Reynolds number used to build the initial POD subspace, and $R e_{2}$ the Reynolds number associated to the new desired dynamic. All the simulations (ROM and DNS in figure 24) are performed at $R e_{2}$. After few DNS iterations, a new snapshot is available. To build a new POD subspace, we have to update the database (§5.2.1), to compute the correlations tensor ( 55.2 .2$)$ and to compute the new POD basis (§5.2.3). All the coefficients of the ROM (21), i.e. $L^{(m)}, L^{(r)}, B^{(m)}$, $B^{(c)}, C^{(m)}$, are built using the current POD basis (corresponding to the modified database), and the integration is performed using $R e \equiv R e_{2}$. Indeed, 
the Reynolds number $R e$ is required to evaluate $B^{(m)}$ (see(16b)). In order to obtain a "real time" POD basis actualization, efficient methods are developed for each step.

\subsubsection{Database modification}

Following equation (7) each snapshot included in the POD database can be approximated by:

$$
\widehat{\boldsymbol{U}}^{\left[1, \cdots, N_{r}\right]}\left(\boldsymbol{x}, t_{k}\right)=\sum_{n=1}^{N_{r}} a_{n}\left(t_{k}\right) \boldsymbol{\Phi}_{n}(\boldsymbol{x}) .
$$

Using one new snapshot, the POD database is modified by either adding this new snapshot, or by replacing the older snapshot by this new one. The position of the new snapshot in the database is denoted by $s$. The number of snapshots included in the new database is thus $N=\max \left(s, N_{s}\right)$. The snapshot $\boldsymbol{U}_{s}$ can be written as the sum of its projection onto the POD basis plus its orthogonal part as:

$$
\widetilde{\boldsymbol{U}}\left(\boldsymbol{x}, t_{s}\right)^{\left[1, \cdots, N_{r}\right]}=\widehat{\boldsymbol{U}}^{\left[1, \cdots, N_{r}\right]}\left(\boldsymbol{x}, t_{s}\right)+\boldsymbol{U}_{s}^{\perp}\left(\boldsymbol{x}, t_{s}\right) .
$$

Therefore, in general, each snapshot is:

$$
\widetilde{\boldsymbol{U}}\left(\boldsymbol{x}, t_{k}\right)^{\left[1, \cdots, N_{r}\right]}=\widehat{\boldsymbol{U}}^{\left[1, \cdots, N_{r}\right]}\left(\boldsymbol{x}, t_{k}\right)+\delta_{k s} \boldsymbol{U}^{\perp}\left(\boldsymbol{x}, t_{s}\right),
$$

where $\delta$ represents the Kronecker symbol.

\subsubsection{Evaluation of the temporal correlation tensor}

In order to reduce the computational costs, the temporal correlation tensor $C$ is calculated using the snapshots decomposition $\widetilde{\boldsymbol{U}}_{k}^{\left[1, \cdots, N_{r}\right]}$.

$$
\begin{aligned}
C\left(t_{k}, t_{l}\right) & =\left(\widetilde{\boldsymbol{U}}\left(\boldsymbol{x}, t_{k}\right)^{\left[1, \cdots, N_{r}\right]}, \widetilde{\boldsymbol{U}}\left(\boldsymbol{x}, t_{l}\right)^{\left[1, \cdots, N_{r}\right]}\right)_{\Omega} \\
& =\left(\sum_{i=1}^{N_{r}} a_{i}\left(t_{k}\right) \boldsymbol{\phi}_{i}(\boldsymbol{x})+\delta_{k s} \boldsymbol{U}^{\perp}\left(\boldsymbol{x}, t_{k}\right), \sum_{j=1}^{N_{r}} a_{j}\left(t_{l}\right) \boldsymbol{\phi}_{j}(\boldsymbol{x})+\delta_{l s} \boldsymbol{U}^{\perp}\left(\boldsymbol{x}, t_{l}\right)\right)_{\Omega} \\
& =\sum_{i=1}^{N_{r}} \sum_{j=1}^{N_{r}} a_{i}\left(t_{k}\right) a_{j}\left(t_{l}\right) \underbrace{\left(\boldsymbol{\phi}_{i}(\boldsymbol{x}), \boldsymbol{\phi}_{j}(\boldsymbol{x})\right)_{\Omega}}_{=\delta_{i j}}+\delta_{k s} \delta_{l s}\left(\boldsymbol{U}^{\perp}\left(\boldsymbol{x}, t_{k}\right), \boldsymbol{U}^{\perp}\left(\boldsymbol{x}, t_{l}\right)\right)_{\Omega} \\
& +\delta_{l s} \sum_{i=1}^{N_{r}} a_{i}\left(t_{k}\right) \underbrace{\left(\boldsymbol{\phi}_{i}(\boldsymbol{x}), \boldsymbol{U}^{\perp}\left(\boldsymbol{x}, t_{l}\right)\right)_{\Omega}}_{=0}+\delta_{k s} \sum_{j=1}^{N_{r}} a_{j}\left(t_{l}\right) \underbrace{\left(\boldsymbol{U}^{\perp^{T}}\left(\boldsymbol{x}, t_{k}\right) \boldsymbol{\phi}_{j}(\boldsymbol{x})\right)_{\Omega}}_{=0} .
\end{aligned}
$$

Hence, the approximation of the temporal correlation tensor simply writes:

$$
C\left(t_{k}, t_{l}\right)=\sum_{i=1}^{N_{r}} a_{i}\left(t_{k}\right) a_{i}\left(t_{l}\right)+\delta_{k s} \delta_{l s} \int_{\Omega} \sum_{i=1}^{n_{c}} U^{\perp^{i}}\left(\boldsymbol{x}, t_{k}\right) U^{\perp^{i}}\left(\boldsymbol{x}, t_{l}\right) \mathrm{d} \boldsymbol{x} .
$$


The evaluation of this matrix is very fast. Indeed, it is not necessary to calculate snapshots correlations on the whole mesh, but only correlations on the retained temporal coefficients. Other than that, it is only necessary to evaluate one auto-correlation with $\boldsymbol{U}_{s}^{\perp}$ to evaluate the component $C_{s s}$.

\subsubsection{Actualization of the POD basis}

All the quantities evaluated before the calculation of the temporal correlation tensor (33) are superscripted by $(n)$. The actualized POD basis could be evaluated from (see [3] for more details):

$$
\begin{aligned}
& \boldsymbol{\phi}_{k}^{(n+1)}(\boldsymbol{x})=\frac{1}{\lambda_{k}^{(n+1)}} \sum_{j=1}^{N_{r}} \widetilde{\boldsymbol{U}}^{(n)}\left(\boldsymbol{x}, t_{j}\right) a_{k}^{(n+1)}\left(t_{j}\right) \\
& =\frac{1}{\lambda_{k}^{(n+1)}} \sum_{j=1}^{N_{r}}\left(\sum_{i=1}^{N_{r}} a_{i}^{(n)}\left(t_{j}\right) \phi_{i}^{(n)}(\boldsymbol{x})+\delta_{j s} \boldsymbol{U}^{\perp}(n)\left(\boldsymbol{x}, t_{j}\right)\right) a_{k}^{(n+1)}\left(t_{j}\right) \\
& =\frac{1}{\lambda_{k}^{(n+1)}} \sum_{i=1}^{N_{r}} \sum_{j=1}^{N_{r}} a_{k}^{(n+1)}\left(t_{j}\right) a_{i}^{(n)}\left(t_{j}\right) \phi_{i}^{(n)}(\boldsymbol{x})+\frac{1}{\lambda_{k}^{(n+1)}} \boldsymbol{U}^{\perp}{ }^{(n)}\left(\boldsymbol{x}, t_{s}\right) a_{k}^{(n+1)}\left(t_{s}\right) \text {. }
\end{aligned}
$$

Introducing the modal correlation tensor $K^{(n+1)} \in \mathbb{R}^{N_{s} \times N_{s}}$ between old and new time-dependent POD eigenfunctions,

$$
K_{k i}^{(n+1)}=\frac{1}{\lambda_{k}^{(n+1)}} \sum_{j=1}^{N_{r}} a_{k}^{(n+1)}\left(t_{j}\right) a_{i}^{(n)}\left(t_{j}\right),
$$

and the vector

$$
\boldsymbol{S}_{k}^{(n+1)}(\boldsymbol{x})=\frac{1}{\lambda_{k}^{(n+1)}} \boldsymbol{U}^{\perp(n)}\left(\boldsymbol{x}, t_{s}\right) a_{k}^{(n+1)}\left(t_{s}\right),
$$

we have:

$$
\boldsymbol{\phi}_{k}^{(n+1)}(\boldsymbol{x})=\sum_{i=1}^{N_{r}} K_{k i}^{(n+1)} \boldsymbol{\phi}_{i}^{(n)}(\boldsymbol{x})+\boldsymbol{S}_{k}^{(n+1)}(\boldsymbol{x}) .
$$

Using the matrix $S^{(n+1)}$ with elements $S_{i j}^{(n+1)}=S_{i}^{j(n+1)}$, the new POD basis can be obtained from the old one using the linear application $\varphi: \mathbb{R}^{n} \times \mathbb{R}^{n} \mapsto$ $\mathbb{R}^{n} \times \mathbb{R}^{n}$ defined as follows:

$$
\varphi: \phi^{(n)} \mapsto \phi^{(n+1)}=\phi^{(n)} K^{(n+1)}+S^{(n+1)} .
$$

The actualization of the POD basis is thus much faster than in the classical way (reconstruction of a new POD basis). Indeed, it is not necessary to make a sum on the whole set of snapshots $(N)$, but only to make a weighted sum on the $N_{r} \ll N$ old POD modes plus a part of the orthogonal contribution.

This process for the hybrid method can be applied at each new snapshot. 


\subsubsection{Numerical results}

During the simulation of the confined square cylinder wake flow at $R e_{1}=100$, 40 snapshots are uniformly taken over a vortex shedding period to evaluate the temporal correlation tensor.

In the first phase, the method to evaluate the temporal correlation tensor introduced in $§ 5.2 .2$ is tested. A comparison between eigenvalues of the temporal correlation tensor evaluated from the exact field $\boldsymbol{U}$ and from a $N_{r}$-mode approximated one, $\widetilde{\boldsymbol{U}}^{\left[1, \cdots, N_{r}\right]}$, is presented in Fig. (25). For this example we set $N_{r}=5$ and $N_{r}=11$. In the two cases, the Relative Information Content $R I C\left(N_{r}\right)$ is greater than $99 \%$. It can be seen that the $N_{r}$ first eigenvalues computed from the $N_{r}$-approximated temporal correlation tensor accurately fit the eigenvalues computed from the exact temporal correlation tensor. All the other approximated eigenvalues for $n>N_{r}$ are equal to zero except $\lambda_{N_{r}+1}$ which indicates a non-zero contribution of the orthogonal part $\boldsymbol{U}^{\perp}$.

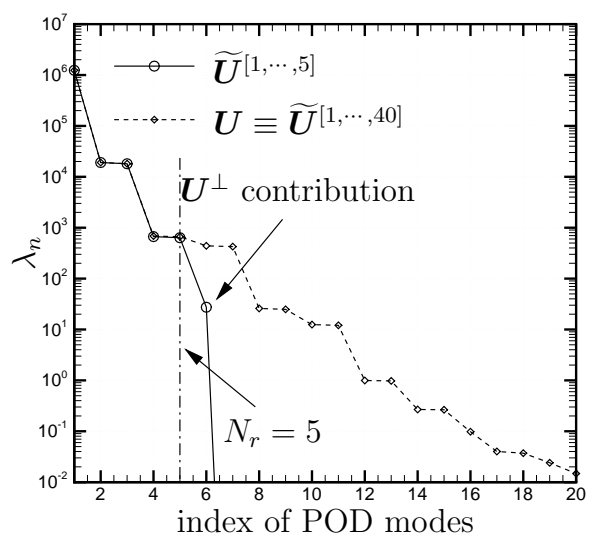

(a) $N_{r}=5$.

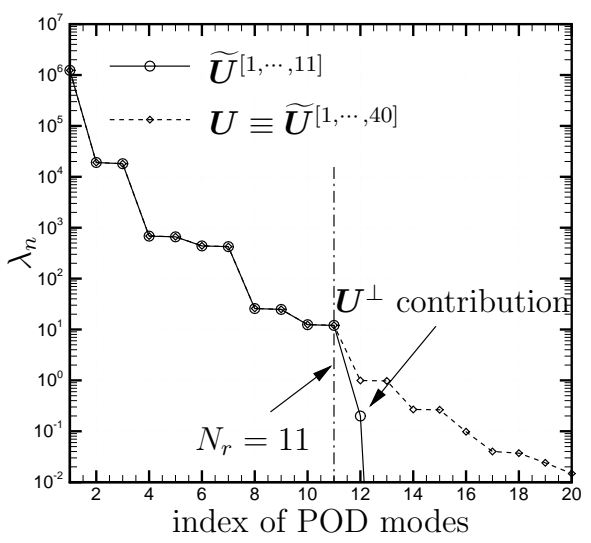

(b) $N_{r}=11$.

Fig. 25. Comparison of the temporal correlation tensor eigenvalues evaluated from the exact field, $\boldsymbol{U}$, and from the $N_{r}$-modes approximated one, $\widetilde{\boldsymbol{U}}^{\left[1, \cdots, N_{r}\right]}$.

Then, the influence of the linear actualization of the POD basis is considered. Results of the linear actualization of the POD basis functions introduced in section 5.2.3 are presented in Fig. 26. Here, one step of the actualization of a transient flow from $R e_{1}=100$ to $R e_{2}=200$ is presented. As in the previous illustration, a 40 snapshots database is used to compute the initial POD basis $\phi^{(0)}$.

We consider the results concerning the hybrid method. The initial and target POD basis, $\boldsymbol{\Phi}^{R e_{1}}$ and $\boldsymbol{\Phi}^{R e_{2}}$, correspond to Reynolds numbers $R e_{1}=100$ and $R e_{2}=200$ respectively. Figure 27 shows the evolution of the convergence criterion $\boldsymbol{\Phi} \cdot \boldsymbol{\Phi}^{R e_{2}}$ versus the number of vortex shedding periods (and so versus the number of actualization iterations) for different percentages of actual the 


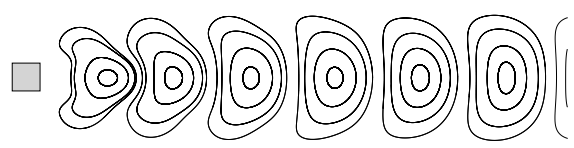

(a) $\phi_{2}^{(0)}$.

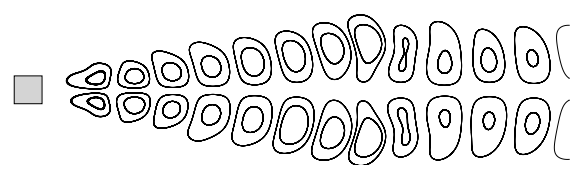

(c) $\phi_{4}^{(0)}$.

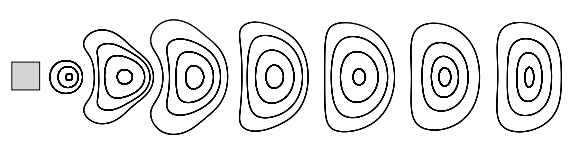

(b) $\phi_{2}^{(1)}=\varphi\left(\left\{\phi_{i}^{(0)}\right\}_{i=1}^{N}, \boldsymbol{U}^{\perp}\right)$.

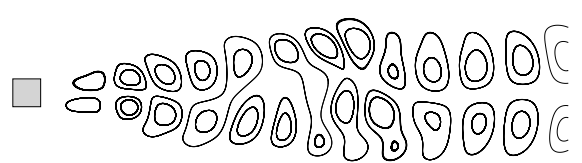

(d) $\phi_{4}^{(1)}=\varphi\left(\left\{\boldsymbol{\phi}_{i}^{(0)}\right\}_{i=1}^{N}, \boldsymbol{U}^{\perp}\right)$.

Fig. 26. Modification of the POD basis functions under the application of the linear transfomation $\varphi$. Streamline representation of the velocity fields.

DNS. Denoting $T_{N S}$ and $T_{R O M}$ the time intervals where we use either DNS or POD ROM ${ }^{7}$ respectively, the percentage of DNS is defined as $P_{N S}=$ $T_{N S} /\left(T_{N S}+T_{R O M}\right)$.

The simulation with $P_{N S}=100 \%$ does not involve any ROM. It can be seen that 10 vortex shedding periods are necessary to converge towards the target POD basis using only DNS. This time corresponds to that required for perturbations, coming from the dynamics changes $R e_{1} \rightarrow R e_{2}$, to cross the whole simulation domain $\Omega$ and to reach an asymptotic behavior. The same results can approximatively be obtained with $P_{N S}=90 \%, P_{N S}=80 \%$ and with less accuracy $70 \%$ DNS. However, no convergence is obtained with less than $P_{N S}=70 \%$. Hence, a sufficient amount of DNS is necessary to converge toward the target POD basis. An explanation of this phenomenon is given in figure 28. Since the POD basis is not well adapted to give a good representation of the current flow (there is a delay in the adaptation process), the solution of the POD ROM moves away from the exact "desired" solution. The DNS has to be able to correct this drift. If the DNS is able to correct the error made by the POD ROM (see figures 28(a) and 28(b)), the convergence is obtained. However, if the DNS is not able to correct this error (see figure 28(c)), it is not possible to converge toward the target basis.

A few comments about the numerical costs can be given. The computational cost due to the ROM is negligible in comparison with the DNS ones. Also, the POD basis update described in sections 5.2.1, 5.2.2 and 5.2.3 generates very low numerical costs. In practice, our estimation is that taking $P_{N S}=70 \%$, which still gives good results in terms of approximation, approximatively $20 \%$ of the total numerical costs can be saved as compared to DNS.

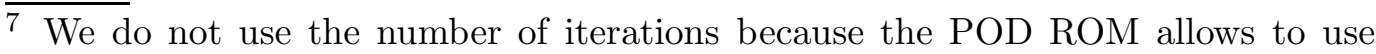
greater time steps than the DNS.
} 


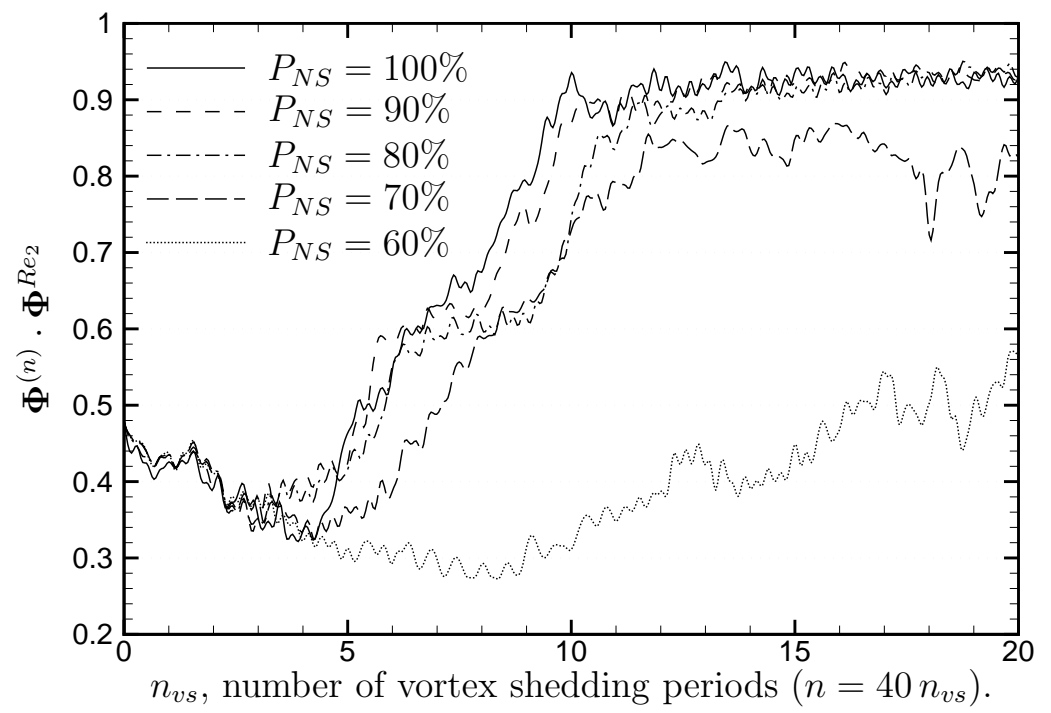

Fig. 27. Evolution of the convergence criterion versus the number of vortex shedding periods.

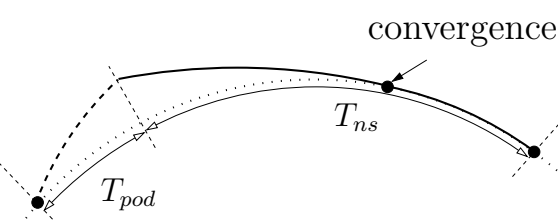

(a) $P_{N S}>70 \%$.

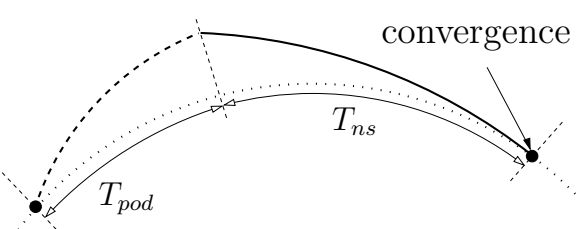

(b) $P_{N S}=70 \%$.

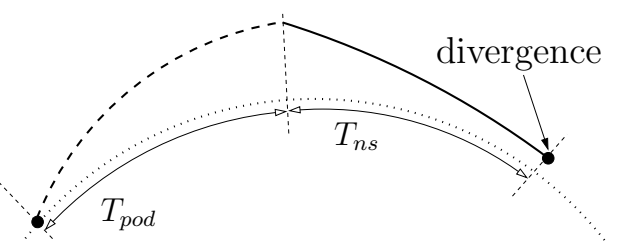

(c) $P_{N S}<70 \%$.

Fig. 28. Schematic explanation for the convergence of the hybrid DNS-POD method. -... exact "target"; - - - POD ROM ; DNS. 


\section{CONCLUSIONS}

The objective of this paper was to improve reduced order modeling based on proper orthogonal decomposition. Indeed, the proper orthogonal decomposition method is a viable technique to build low dimensional models, but it also presents several drawbacks: (i) since usually only velocity fields are used to build the reduced order model, the pressure term has to be modeled, (ii) it is necessary to model the effects of the fine scale that are not explicitly taken into account in the expansion and that are responsible of the main part of the viscous dissipation, and (iii) the POD basis functions are only able to give a good representation of the flow dynamics included in the given snapshots database.

We have shown how to build a pressure extended reduced order model at no additional cost in comparison with a standard velocity ROM. Using this pressure extended ROM, it is not necessary to model the pressure term. Since both the velocity and pressure POD fields are available, it is possible to evaluate the Navier-Stokes operator residuals using these POD fields. Although this model gives very good results in term of asymptotic solution, it is still necessary to model the effects of the unresolved fines scales.

In this respect, different stabilization methods for the reduced order model were taken under consideration. The stabilization we propose consists in modeling the effect of the missing fine scales. To this end, we made the choice of using residuals of Navier-Stokes operator evaluated from POD fields. The first method proposed consists in enlarging the POD subspace with few residuals modes. If the original number of modes is not large enough to provide appropriate dissipation, this method is able to stabilize the model. The stabilization is not due to the enlargement of the POD subspace, in fact an unstable model can be stabilized replacing a few number of original POD modes with the same number of residual modes. No empiric parameter has to be estimated in this approach. The second approach proposed relies on an approximation of the fine scale equation. Both SUPG and VMS methods give good results. In this approach, only two parameters have to be estimated. In some specific cases [39], these parameters can be approximated using some scaling arguments, leading to an universal model with no empiricism.

Finally, we have tried to improve the POD functional subspace. The goal was to derive efficient methods to adapt the POD basis when dynamics changes (with control parameters). The first method is a Krylov-like method. This method is based on iteratively including in the basis some POD-NS residuals (this is an iterative version of the first stabilization method). When the size of the basis becomes too large, a new POD compression is performed. This method gives very good results for the $1 D$ Burgers equation, but convergence 
is too slow for the $2 D$ Navier-Stokes equations, at least in our configuration. One explanation is that the "missing" scales (when dynamics evolves) are not necessary "fine" scales, and thus the approximation using POD-NS residuals is not good. The second method is an hybrid method that couples DNS and reduced order models. The idea is to modify and update the database when dynamical evolution occurs, so that the leading basis functions always represent the updated dynamics. This method, coupled with a fast method to actualize the POD basis functions using this new database, gives good results if a sufficient amount of DNS is performed. Approximatively $20 \%$ of the total numerical costs can be saved using such hybrid method.

\section{References}

[1] T. Bui-Thanh, K. Willcox, O. Ghattas, B. van Bloemen Waander, Goaloriented, model-constrained optimization for reduction of large-scale systems, J. Comp. Phys. 224 (2) (2007) 880-896.

[2] P. Holmes, J. L. Lumley, G. Berkooz, Turbulence, Coherent Structures, Dynamical Systems and Symmetry, Cambridge Monographs on Mechanics, 1996.

[3] L. Sirovich, Turbulence and the dynamics of coherent structures, Quarterly of Applied Mathematics XLV (3) (1987) 561-590.

[4] L. Cordier, M. Bergmann, Proper Orthogonal Decomposition: an overview, in: Lecture series 2002-04 on post-processing of experimental and numerical data, Von Kármán Institute for Fluid Dynamics, 2002.

[5] J. Nocedal, S. J. Wright, Numerical Optimization, Springer series in operations research, 1999.

[6] C. W. Rowley, Model reduction for fluids, using balanced proper orthogonal decomposition, Int. J. on Bifurcation and Chaos 15 (3) (2005) 997-1013.

[7] K. Willcox, J. Peraire, Balanced model reduction via the proper orthogonal decomposition, AIAA Journal 40 (11) (2002) 2323-2330.

[8] E. Akervik, G. Hœpffner, U. Ehrenstein, D. S. Henningson, Optimal growth, model reduction and control in a separated boundary-layer flow using global eigenmodes, J. Fluid Mech. 579 (2007) 305-314.

[9] B. R. Noack, H. Eckelman, A low dimensional galerkin method for the threedimensinal flow around a circular cylinder, Phys. Fuilds 6 (1) (1994) 124-143.

[10] D. Rempfer, On low-dimensional Galerkin models for fluid flow, Theor. Comput. Fluid Dyn. 14 (2000) 75-88. 
[11] B. R. Noack, P. Papas, P. A. Monkewitz, The need for a pressure-term representation in empirical galerkin models of incompressible shear-flows, J. Fluid Mech. 523 (2005) 339-365.

[12] B. Galletti, C.-H. Bruneau, L. Zannetti, A. Iollo, Low-order modelling of laminar flow regimes past a confined square cylinder, J. Fluid Mech. 503 (2004) $161-170$.

[13] P. Sagaut, Large-eddy simulation for incompressible flows - An introduction, Springer-Verlag, 2005.

[14] Y. Bazilevs, V. M. Calo, J. A. Cottrell, T. J. R. Hugues, A. Reali, G. Scovazzi, Variational multiscale residual-based turbulence modeling for large eddy simulation of incompressible flows, Comput. Methods Appl. Mech. Engrg. 197 (2007) 173-201.

[15] R. D. Prabhu, S. S. Collis, Y. Chang, The influence of control on Proper Orthogonal Decomposition of wall-bounded turbulent flows, Phys. Fluids 13 (2) (2001) 520-537.

[16] B. R. Noack, K. Afanasiev, M. Morzyński, G. Tadmor, F. Thiele, A hierarchy of low-dimensional models for the transient and post-transient cylinder wake, J. Fluid Mech. 497 (2003) 335-363.

[17] M. Bergmann, L. Cordier, Optimal control of the cylinder wake in the laminar regime by trust-region methods and pod reduced-order models, J. Comp. Phys. 227 (16) (2008) 7813-7840.

[18] J. L. Lumley, Atmospheric Turbulence and Wave Propagation. The structure of inhomogeneous turbulence, A.M. Yaglom \& V.I. Tatarski, 1967, pp. 166-178.

[19] L. Cordier, M. Bergmann, Two typical applications of POD: coherent structures eduction and reduced order modelling, in: Lecture series 2002-04 on postprocessing of experimental and numerical data, Von Kármán Institute for Fluid Dynamics, 2002.

[20] S. Sirisup, G. E. Karniadakis, A spectral viscosity method for correcting the long-term behavior of POD model, J. Comp. Phys. 194 (2004) 92-116.

[21] A. E. Deane, I. G. Kevrekidis, G. E. Karniadakis, S. A. Orszag, Low-dimensional models for complex geometry flows: Application to grooved channels and circular cylinders, Phys. Fluids 3 (10) (1991) 2337-2354.

[22] M. Bergmann, Optimisation aérodynamique par réduction de modèle POD et contrôle optimal. Application au sillage laminaire d'un cylindre circulaire., Ph.D. thesis, Institut National Polytechnique de Lorraine, Nancy, France (2004).

[23] N. Aubry, P. Holmes, J. L. Lumley, E. Stone, The dynamics of coherent structures in the wall region of a turbulent boundary layer, J. Fluid Mech. 192 (1988) 115-173. 
[24] B. Podvin, J. Lumley, A low-dimensional approach for the minimal flow unit, J. Fluid Mech. 362 (1998) 121-151.

[25] J. Delville, L. Ukeiley, L. Cordier, J.-P. Bonnet, M. Glauser, Examination of large-scale structures in a turbulent mixing layer. Part 1. Proper Orthogonal Decomposition, J. Fluid Mech. 391 (1999) 91-122.

[26] D. Rempfer, H. F. Fasel, Evolution of three-dimensional coherent structures in a flat-plate boundary layer, J. Fluid Mech. 260 (1994) 351-375.

[27] D. Rempfer, Investigations of boundary layer transition via Galerkin Projections on Empirical Eigenfunctions, Phys. Fluids 8 (1) (1996) 175-188.

[28] G. S. Karamanos, G. E. Karniadakis, A spectral vanishing viscosity method for Large Eddy Simulations, J. Comp. Phys. 162 (2000) 22-50.

[29] M. Bergmann, L. Cordier, J.-P. Brancher, Optimal rotary control of the cylinder wake using POD Reduced Order Model, Phys. Fluids 17 (9) (2005) 097101:1-21.

[30] M. Buffoni, S. Camarri, A. Iollo, M. Salvetti, Low-dimensional modelling of a confined three-dimensional wake flow, J. Fluid Mech. 569 (2006) 141-150.

[31] M. Couplet, C. Basdevant, P. Sagaut, Calibrated reduced-order POD-Galerkin system for fluid flow modelling, J. Comp. Phys. 207 (2005) 192-220.

[32] V. L. Kalb, A. E. Deane, An intrinsic stabilization scheme for proper orthogonal decomposition based low-dimensional models, Phys. Fuilds 19 (2007) 054106.

[33] W. Cazemier, Proper Orthogonal Decomposition and low-dimensionnal models for turbulent flows, Ph.D. thesis, université de Groningen (1997).

[34] W. Cazemier, R. W. C. P. Verstappen, A. E. P. Veldman, Proper Orthogonal Decomposition and low-dimensional models for driven cavity flows, Phys. Fluids 10 (7) (1998) 1685-1699.

[35] B. R. Noack, M. Schlegel, B. Ahlborn, G. Mutschke, M. Morzynski, P. Comte, G. Tadmor, A finite-time thermodynamics formalism for unsteady flows, J. Non-Equilib. Thermodyn. 3 (2) (2008) 103-148.

[36] G. Vigo, The Proper Orthogonal Decomposition applied to unsteady compressible Navier-Stokes equation, Tech. Rep. 3945, INRIA (1998).

[37] A. Iollo, A. Dervieux, J. A. Désiéri, S. Lanteri, Two stable pod-based approximations to the navier-stokes equations, Comput. Visual. Sci. 3 (2000) 61-66.

[38] A. Iollo, S. Lanteri, J. A. Désiéri, Stability properties of pod-galerkin approximations for the compressible navier-stokes equations, Theoret. Comput. Fluid Dynamics 13 (2000) 377-393.

[39] T. J. R. Hugues, G. Feijóo, L. Mazzei, J. B. Quincy, The variational multiscale method-a paradigm for computational mechanics, Comput. Methods Appl. Mech. Engrg. 166 (1998) 3-24. 
[40] K. Ito, S. S. Ravindran, A reduced-order method for simulation and control of fluid flows, J. Comp. Phys. 143 (1998) 403-425.

[41] S. S. Ravindran, A reduced-order approach for optimal control of fluids using Proper Orthogonal Decomposition, Int. J. Numer. Meth. Fluids 34 (2000) 425448.

[42] W. R. Graham, J. Peraire, K. T. Tang, Optimal Control of Vortex Shedding Using Low Order Models. Part 2: Model-based control, Int. J. for Numer. Meth. in Engrg. 44 (7) (1999b) 973-990.

[43] X. Ma, G. E. Karniadakis, A low-dimensional model for simulating threedimensional cylinder flow, J. Fluid Mech. 458 (2002) 181-190.

[44] J. Burkardt, M. D. Gunzburger, H.-C. Lee, Centroidal Voronoi TessellationBased Reduced-Order Modeling of Complex Systems, Tech. rep., Florida State University (2004).

[45] W. R. Graham, J. Peraire, K. T. Tang, Optimal Control of Vortex Shedding Using Low Order Models. Part 1. Open-Loop Model Development, Int. J. for Numer. Meth. in Engrg. 44 (7) (1999a) 945-972.

[46] M. Fahl, Trust-region methods for flow control based on Reduced Order Modeling, Ph.D. thesis, Trier university (2000).

[47] E. Arian, M. Fahl, E. W. Sachs, Trust-Region Proper Orthogonal Decomposition for Flow Control, Icase report 2000-25.

[48] Y. Saad, M. H. Schultz, Gmres : A generalized residual algorithm for solving nonsymetric linear systems, SIAM J. Sci. Stat. Comput. 7 (3) (1986) 856-869.

[49] S. Sirisup, G. E. Karniadakis, D. Xiu, I. G. Kevrekidis, Equation-free/galerkinfree pod-assisted computation of incompressible flows, J. Comp. Phys. 207 (2005) 568-587. 